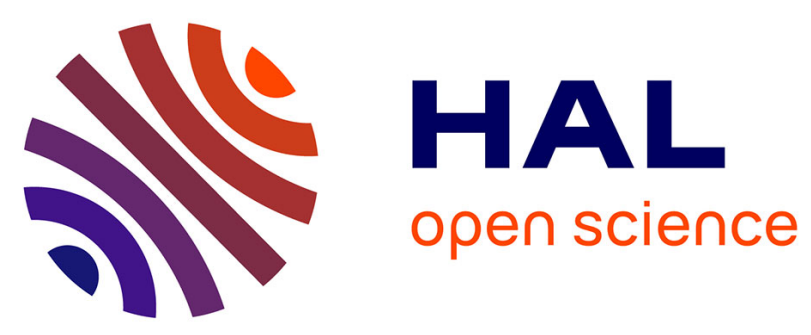

\title{
Iconic Plurality
}

Philippe Schlenker, Jonathan Lamberton

\section{- To cite this version:}

Philippe Schlenker, Jonathan Lamberton. Iconic Plurality. Linguistics and Philosophy, 2019, 42 (1), pp.45-108. 10.1007/s10988-018-9236-0 . hal-03052001

\section{HAL Id: hal-03052001 https://hal.science/hal-03052001}

Submitted on 10 Dec 2020

HAL is a multi-disciplinary open access archive for the deposit and dissemination of scientific research documents, whether they are published or not. The documents may come from teaching and research institutions in France or abroad, or from public or private research centers.
L'archive ouverte pluridisciplinaire HAL, est destinée au dépôt et à la diffusion de documents scientifiques de niveau recherche, publiés ou non, émanant des établissements d'enseignement et de recherche français ou étrangers, des laboratoires publics ou privés. 


\title{
Iconic Plurality*
}

\author{
Philippe Schlenker** \\ (Institut Jean-Nicod, CNRS; New York University) \\ Jonathan Lamberton
}

Linguistics \& Philosophy 2019

Links corrected, November 28, 2019

\begin{abstract}
ASL (American Sign Language) can express plurals by repeating a noun, in an unpunctuated fashion, in different parts of signing space. We argue that this construction may come with a rich (and at-issue) iconic component: the geometric arrangement of the repetitions provides information about the arrangement of the denoted plurality; in addition, the number and speed of the repetitions provide information about the size of the denoted plurality. Interestingly, the shape of the repetitions may introduce a new singular discourse referent when a vertex can be inferred to denote a singular object. Thus one may point towards the first or last iteration of a horizontal repetition of $B O O K$ to denote the left- or right-edge of the corresponding row. This yields a remarkable interaction between iconic semantics and standard logical semantics. We show that our analysis extends to 'punctuated' repetitions, which involve clearly individuated iterations of a singular noun. While these may initially look like coordinated indefinites, they are better handled by the same iconic framework as plural, unpunctuated repetitions. Some repetition-based mass terms also give rise to iconic effects, and to different readings depending on whether the repetition is continuous, unpunctuated, or punctuated. Our analysis highlights the need for a formal semantics with iconicity to study the integration of such iconic and logical conditions. It also raises a question: can similar facts be found in spoken language when gestures are taken into account? We suggest that several effects can be replicated, especially when one considers examples involving 'pro-speech gestures' (= gestures that fully replace some spoken expressions).
\end{abstract}

Keywords: sign language semantics, iconicity, plurals, mass terms, unpunctuated repetitions, punctuated repetitions, continuous repetitions

\footnotetext{
* Special thanks to Sam Alxatib, Emmanuel Chemla, Masha Esipova, Jeremy Kuhn, Salvador Mascarenhas, Rob Pasternak, Benjamin Spector, Brent Strickland and Lyn Tieu for helpful remarks on this research, and to Brian Buccola for discussion of an English sentence of interest. We are also grateful to Helen Koulidobrova for sending us her manuscript on 'Counting Nouns in ASL' (we received it after the present piece was almost finalized, so systematic comparisons are left for the future). We greatly benefited from the remarkably constructive comments of Malte Zimmermann and three anonymous reviewers (Malte Zimmermann's suggestions lead to an important improvement of the final analysis). Many thanks to Lucie Ravaux for preparing the bibliography and checking averages.

Authors' contributions: Philippe Schlenker initiated this research, constructed all examples in consultation with Jonathan Lamberton, and developed all the analysis. Jonathan Lamberton was the ASL consultant for the initial phase of the work. When it was written, he provided transcriptions and translations, as well as descriptions of the iconic properties of the signs. Any theoretical discussion among co-authors occurred only after the data were collected and the first version of the article was written, and primarily by email because the authors were not on the same continent. The separation between the two phases of the work was intended to minimize the risk the 'theoretical contamination' of sign language judgments.

Grant acknowledgments: The research leading to these results received funding from the European Research Council under the European Union's Seventh Framework Programme (FP/2007-2013) / ERC Grant Agreement N³24115-FRONTSEM (PI: Schlenker). Research was conducted at Institut d'Etudes Cognitives, Ecole Normale Supérieure - PSL Research University. Institut d'Etudes Cognitives is supported by grants ANR-10LABX-0087 IEC et ANR-10-IDEX-0001-02 PSL*.

** Institut Jean-Nicod (ENS - EHESS - CNRS), Département d'Etudes Cognitives, Ecole Normale Supérieure, Paris, France; PSL Research University ; New York University, New York.
} 


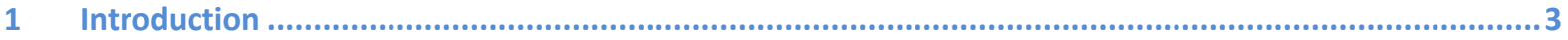

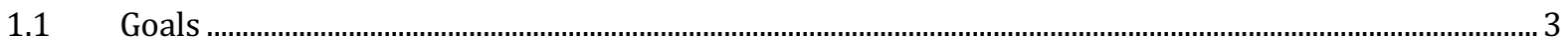

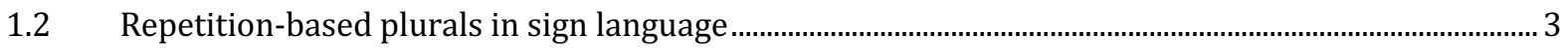

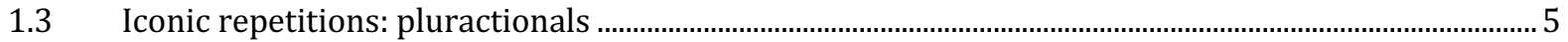

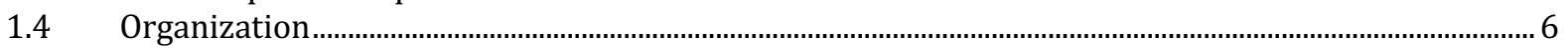

2 Elicitation methods and transcription conventions .......................................................6 6

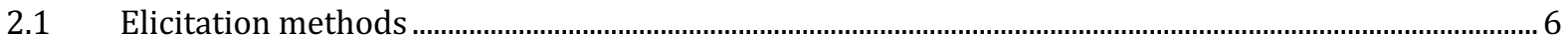

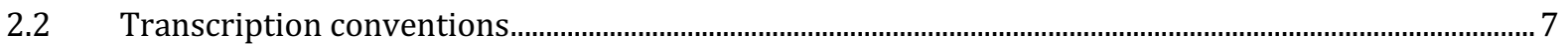

3 Iconic plurals and mass terms in ASL: initial properties ...................................................8

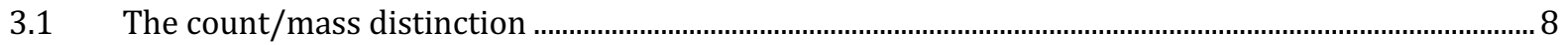

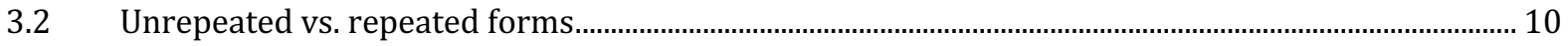

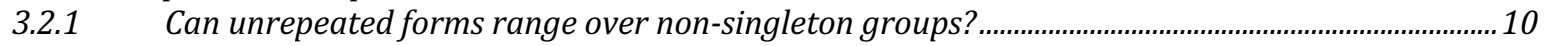

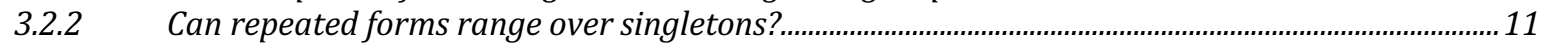

4 Unpunctuated repetitions as plurals: grammatical properties. ........................................... 12

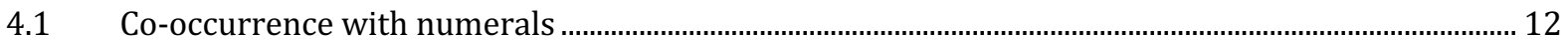

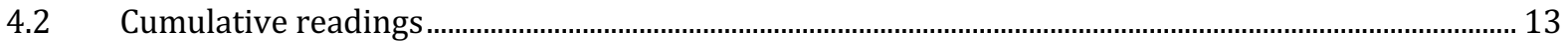

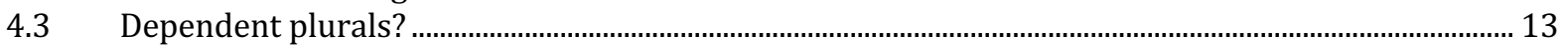

5 Unpunctuated and punctuated repetitions: iconic properties . . . . . . . . . . . . . . . . . . . . . . . . . . . . . . . . . . . . . . . . . 14

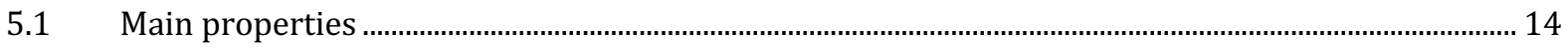

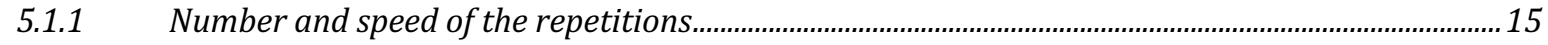

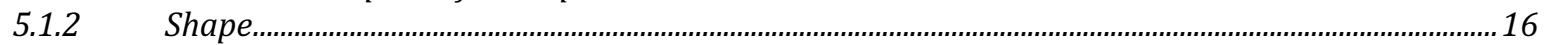

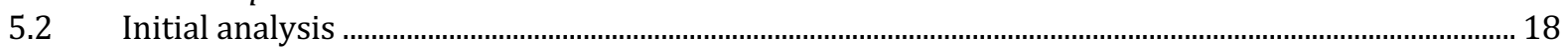

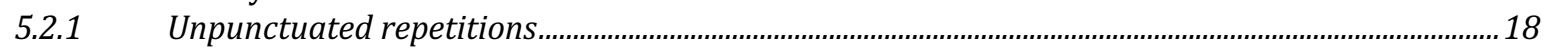

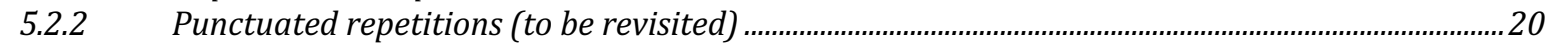

6 Unpunctuated and punctuated repetitions: interaction with anaphora ..................................20

6.1 Singular discourse referents introduced by iconic plurals: the Edge Effect ...................................... 20

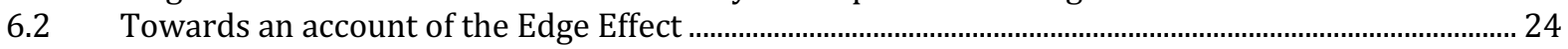

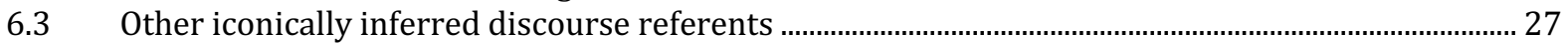

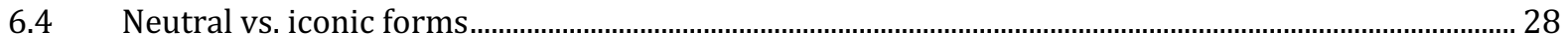

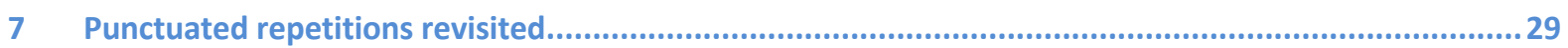

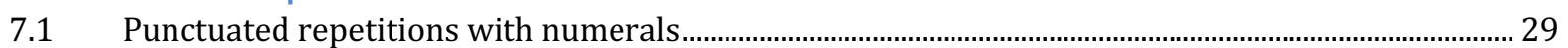

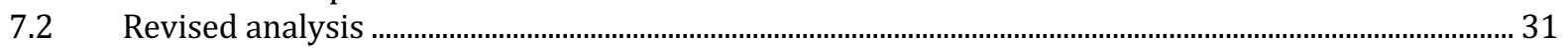

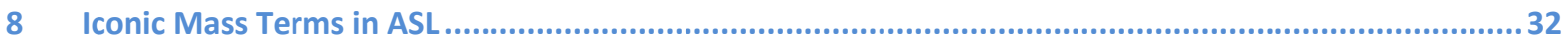

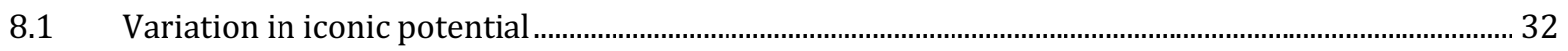

8.2 Types of repetitions: continuous, unpunctuated, punctuated ............................................................ 34

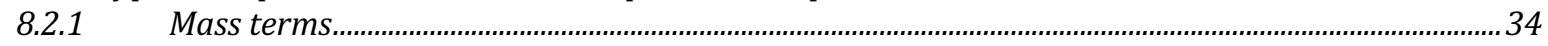

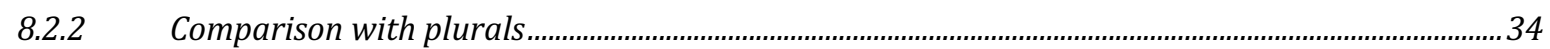

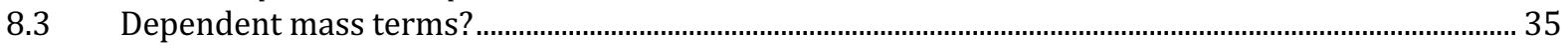

9 Towards a unified account of punctuated, unpunctuated and continuous repetitions ....................36

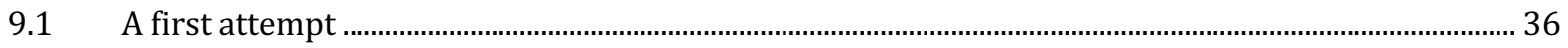

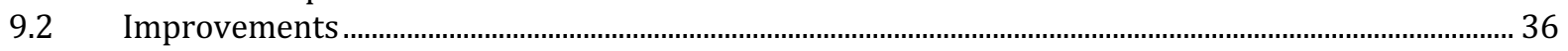

10 Towards a comparison with iconic gestures............................................................... 38

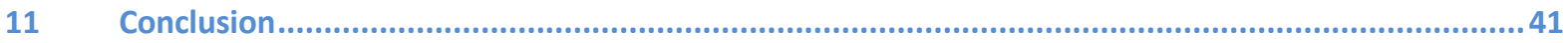

Supplementary Materials: Raw Data...........................................................................................4 43

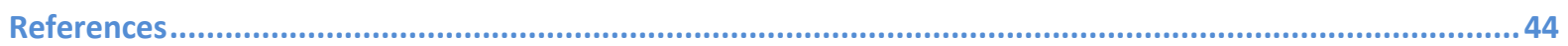




\section{Introduction}

\subsection{Goals}

In sign languages as well as in the expression of some homesigners (deaf individuals who develop a gestural communication system without access to sign language), nominal plurals can be formed by the 'unpunctuated' repetition of a sign (Pfau and Steinbach 2006, Coppola et al. 2013, Abner et al. 2015). In this piece, devoted to American Sign Language (ASL), we will be concerned with constructions in which the noun is repeated in different parts of signing space (the nouns we study do not allow for plural repetition in the same place, although such a mechanism is described in other contexts by Pfau and Steinbach $2006^{1}$ ). A related mechanism of unpunctuated repetition can be applied to verbs to yield pluractional readings (= referring to pluralities of events), and their iconic uses were investigated in Kuhn 2015 and Kuhn and Aristodemo 2017. Nominal unpunctuated repetitions differ in realization from punctuated repetitions. The latter are made of the discrete, clearly separable iteration of the same nominal sign in different parts of signing space; by contrast, unpunctuated repetitions involve iterations with shorter and less distinct breaks between them, which makes these iterations less distinct and sometimes harder to count (similar devices were investigated in homesigners by Coppola et al. 2013 and Abner et al. 2015²). We will also discuss the continuous repetition of a sign, without any break, which is possible with some mass terms.

Our first goal is to show that in ASL, repetition-based plurals based on unpunctuated repetitions with movement may come with a rich iconic component whereby the geometric arrangement of the repeated occurrences provides information about the arrangement of the denoted plurality; the iconic contribution is typically at-issue, which follows a more general pattern for 'iconic modulations' as analyzed in Schlenker to appear d. This iconic effect is also part of a broader pattern in which the number and speed of the repetitions provides information about the size of the denoted plurality. Interestingly, a repetition-based plural may introduce a new singular discourse referent when a vertex of the iconic representation can be inferred to denote a singular object. Thus one may point towards the first or last iteration of a horizontal repetition of $B O O K$ to denote the left- or right-edge of the corresponding row of books. This yields a remarkable interaction between iconic semantics and standard logical semantics.

We will also show that our analysis extends to punctuated repetitions, which as mentioned involve clearly individuated iterations of a singular sign. Due to their discrete character, these repetitions may initially look like coordinated indefinites, but we will argue that they are better handled by the same iconic framework as plural, unpunctuated repetitions. Some repetition-based mass terms also give rise to iconic effects, and to different readings depending on whether the repetition is continuous, unpunctuated, or punctuated - an observation that can be explained by our analysis.

Overall, our observations highlight the need for a formal semantics with iconicity to study the integration of iconic and logical conditions in sign language. They also raise a question: to what extent can analogous iconic facts be found in spoken language when gestures are taken into account? We will suggest that several effects can be replicated within spoken language, especially when one considers examples involving 'pro-speech gestures' (= gestures that fully replace some spoken expressions).

\subsection{Repetition-based plurals in sign language}

In a broad and detailed typological study, Pfau and Steinbach 2006 note that "reduplication and zero marking appear to be two basic pluralization strategies attested in all sign languages that have been investigated so far. Besides simple and sideward reduplication, some sign languages also add

\footnotetext{
${ }^{1}$ See also Fischer 1973 for an early study of reduplication in ASL.

${ }^{2}$ Coppola et al. 2013 describe punctuated repetitions in homesigners as "series of discrete movements, each referring to an entity or action in the vignette. Each movement was clearly articulated and easily segmentable from the rest of the movements." By contrast, unpunctuated repetitions "were movements produced in rapid succession with no clear break between them. Although the pauses between these iterations were much smaller than those separating the components of Punctuated Movements, they were identifiable and could be easily counted. These movements could be produced in a single space, but more often were produced in multiple spatial locations."
} 
(alternating) movement by the non-dominant hand and reduplication of secondary movements to express the plural." As they note, what they call reduplication is really triplication, i.e. the triple iteration of a sign - an observation that will hold in our data as well. Importantly, they find phonological restrictions on reduplication (i.e. triplication) that are "very similar across sign languages": bodyanchored nouns as well as nouns that involve complex movement usually form their plural by way of zero marking; within non-body-anchored nouns, those that involve movement in the midsagittal plane (i.e. the vertical plane that follows the signer's eyegaze if looking forward) form their plural with 'simple reduplication' (in one and the same position), whereas signs that appear in the lateral signing space make use of sideward reduplication (in different parts of space). Pfau and Steinbach also emphasize that in the case of classifiers ${ }^{3}$, sideward reduplication comes with iconic conditions, and they cite remarks about the Sign Language of the Netherlands (NGT) by Nijhof and Zwitserlood 1999 that suggest that iconic conditions are also found with the sideward repetition of normal nouns.

We will be solely concerned with patterns of repetition with movement in ASL, and we will not seek to construct a typology of pluralization strategies, nor of phonological constraints on these operations. But we should still say a word about prior literature pertaining to ASL plurals. Pfau and Steinbach 2006 summarize four generalizations due to Wilbur 1987: first, "if a noun is made with one hand at a location on the face, its plural can be realized by repeating the sign alternately with both hands. Second, whenever a noun makes contact with some body part or involves a change of orientation, the plural is made by reduplication and usually an addition of a horizontal arc path movement. Third, nouns that involve some kind of secondary movement are pluralized without reduplication by continuing the secondary movement (and possibly by adding an additional horizontal sweep). Fourth, (...) nouns that have inherent repetition of movement in their singular form cannot undergo (sideward) reduplication." (see also Abner and Wilbur, to appear for a broader discussion of quantification in ASL). Our claim in the following will be that, in those cases that allow for plural formation by way of repetition with movement, iconic conditions interact in an interesting way with logical semantics and anaphora. ${ }^{4}$

The three types of repetitions we will be investigating are illustrated in (1) (with acceptability judgments on a 7-point scale at the beginning of each example; elicitation methods and transcription conventions are discussed below in Section 2).

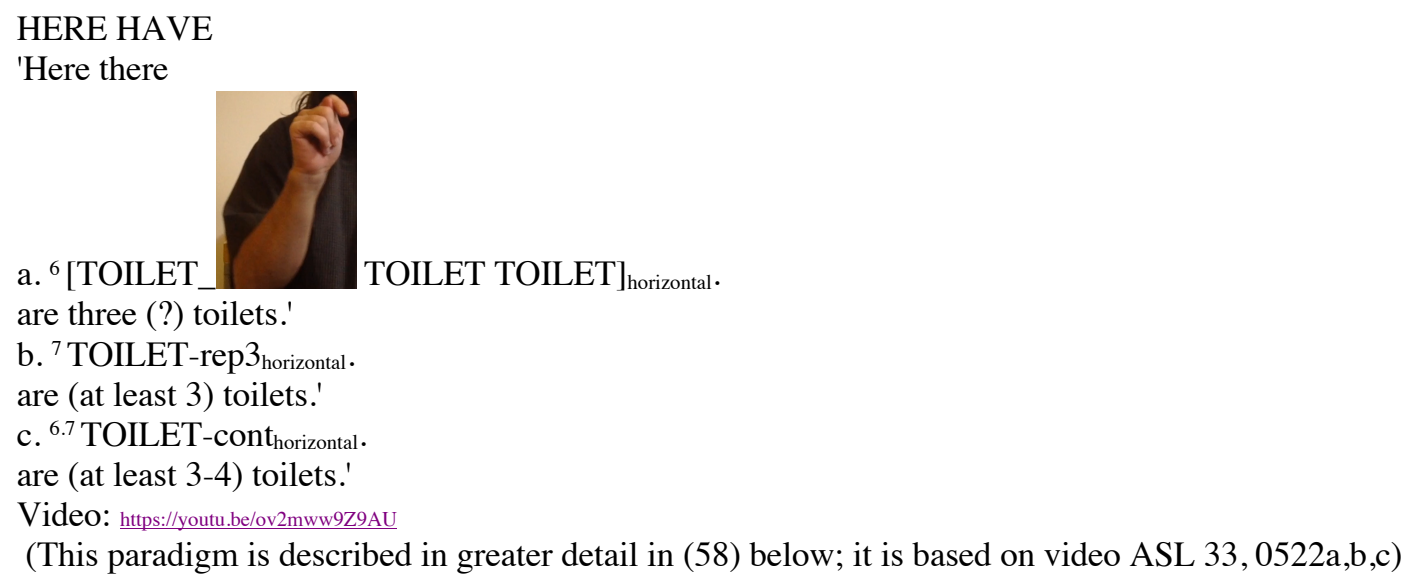

While the fine-grained semantics will be discussed later, we note the main phonetic differences of relevance for our study; a video of the manual part of the signs is included for the reader's convenience (a video sketch is also provided for related contrasts in (28) below). (1)a is a punctuated repetition, with three horizontal iterations of the word TOILET, realized as a trembled $T$ of the manual alphabet, whose first iteration is illustrated (it also comes with a mouthing that distinguishes it from the term for urine, which will make for particularly minimal, if unpoetic, contrasts between these count and mass terms). Characteristic is the fact that the hand goes down between the three iterations, which makes

\footnotetext{
${ }^{3}$ In sign language, classifiers are elements that encode the size, shape and/or type of an object, and enter in highly iconic constructions, in particular ones that encode the movement of the object; see for instance Supalla 1982 and Sandler, Lillo-Martin 2006 and Zwitserlood 2012 for discussion.

${ }^{4}$ Since all our target examples will involve repetition with movement, we will henceforth often drop the modifier.
} 
them easy to individuate: the $T$ handshape disappears or goes back to a neutral position between iterations. In (1)b, there are three iterations as well, but realized a bit faster, and in an unpunctuated fashion, characterized by the fact that the hand only partly goes down between the iterations: the $T$ handshape only partially disappears between them. The realization can be much faster in other examples, making it hard to count the iterations, and highlighting the contrast with punctuated repetitions (which is rather subtle in (1)). Finally, in (1)c a continuous repetition is illustrated; the hand does not go down at all between the iterations, and these are faster, more numerous, and very hard to count.

\subsection{Iconic repetitions: pluractionals ${ }^{5}$}

To our knowledge, there has been little work on the interaction between iconic and logical conditions in nominal repetition-based plurals. By contrast, Kuhn 2015 and Kuhn and Aristodemo 2017 study this interaction in great detail in their study of sign language repetition-based pluractionals (i.e. verbal forms that refer to pluralities of events). In particular, they argue that repetition-based pluractionals in LSF (French Sign Language) give rise to iconic effects, and that these can make at-issue contributions.

As an example, the difference in realization between the 'accelerating' and the 'decelerating' versions of LSF GIVE used in (2)a and (2)b are represented in (3)a and (3)b respectively. The translations immediately show that the rate of production of the iterations of the signs are interpreted: accelerating repetitions of the sign refer to accelerating repetitions of the event, and similarity for decelerating repetitions. Kuhn 2015 and Kuhn and Aristodemo 2017 make specific proposals about the iconic rule of interpretation at work here. Here GIVE-rep-accelerating and GIVE-rep-decelerating transcribe respectively the accelerating and decelerating repetitions of $G I V E^{6}$.

(2) a. MIRKO CHILD BOOK GIVE-rep-accelerating.

'Mirko gave the child a book at an accelerating pace.' (Kuhn and Aristodemo 2017)

b. MIRKO CHILD BOOK GIVE-rep-decelerating.

'Mirko gave the child a book at decelerating pace.' (Kuhn and Aristodemo 2017)

(3) Time-course diagrams of accelerating and decelerating GIVE-rep (Kuhn and Aristodemo 2017)

a. Acceleration
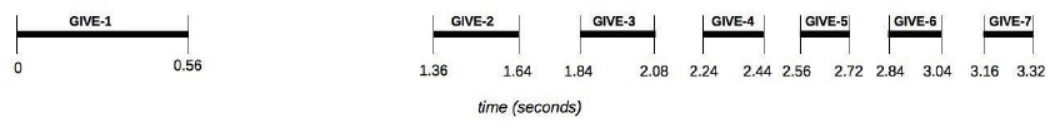

b. Deceleration

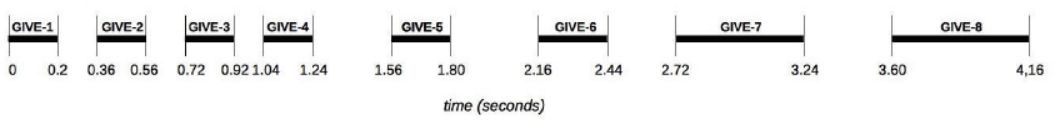

In recent formal approaches, a sign realized with an iconic modulation has often been treated by requiring that its denotation should satisfy analogues of relevant geometric properties of the sign (Schlenker et al. 2013, Schlenker to appear a, b). For example, in the case of iconic pluractional repetition, the faster the repetition of the sign, the faster the repetition of the denoted events (but see Kuhn 2015 and Kuhn and Aristodemo 2017 for a more specific proposal). For the most part, we will be content in this piece to assume an intuitive understanding of iconic conditions, but it should be clear that these should in principle be analyzed within a formal and predictive framework, possibly along the lines of the iconic semantics developed in Greenberg 2013.

Importantly for our purposes, Kuhn and Aristodemo 2017 show that this iconic enrichment can be at-issue and can take scope within a conditional, as seen in (4); here $I X-a$ and $I X-b$ are pointing signs towards positions $a$ and $b$.

\footnotetext{
${ }^{5}$ This paragraph borrows from Schlenker to appear d.

${ }^{6}$ Kuhn and Aristodemo use 'speeding up' as well as 'accelerating' in some of their transcriptions, but they have confirmed that these are synonymous.
} 
(4) SECRETARY IX-a, IF MIRKO PAPERS IX-b GIVE-rep-accelerating, IX-a HAPPY.

'If Mirko gives papers at an accelerating rate, the secretary will be happy.' (LSF, Kuhn and Aristodemo 2017)

Their results extend to ASL - in this case with a slightly different repetition marker, which involves two-handed, alternating repetition (-alt) rather than one-handed repetition (-rep) (both forms exist in ASL and LSF, and there are semantic and distributional differences between them, as discussed in Kuhn and Aristodemo 2017).

(5) IF JOHN PAPERS GIVE-alt-accelerating, SECRETARY WILL HAPPY.

'If John gives papers at an accelerating rate, the secretary will be happy.' (ASL, Kuhn and Aristodemo to appear)

These results will be important for our purposes, since we will show that repetition-based nominal plurals can also make iconic contributions, and that these too can be at-issue. This conclusion falls under a broader generalization, discussed in Schlenker to appear d: in spoken and sign language alike, iconic modulations of words and signs can have an at-issue status - unlike co-speech gestures, for instance, which have been claimed to be non-at-issue, for instance in Ebert and Ebert 2014 and Schlenker to appear c, d.

It should be added that Henderson 2016 investigates pluractional ideophones in some Mayan languages, drawing an explicit connection with the iconic effects of sign language pluractionals discussed by Kuhn and Aristodemo. Henderson does not mention similar effects with nominal plurals, however - and some aspects of his analysis suggest that he takes his spoken language effects to be in principle restricted to constructions that have an event-related semantics.

\subsection{Organization}

The rest of this article is organized as follows. Elicitation and transcription conventions are introduced in Section 2. Initial properties of iconic plurals and mass terms are introduced in Section 3. Grammatical properties of unpunctuated repetitions are described in Section 4. Iconic properties of repetitions, both punctuated and unpunctuated, are discussed in Section 5. Their ability to create new discourse referents on iconic grounds is analyzed in Section 6. Punctuated repetitions are revisited in Section 7, where it is shown that they cannot just be analyzed as coordinated indefinites. We then turn to iconic mass terms in Section 8. A unified account of punctuated, unpunctuated and continuous repetitions is sketched in Section 9, and we speculate in Section 10 that the main properties of all three types of repetitions can be replicated with some iconic gestures in spoken language. Finally, concluding remarks are made in Section 11. Raw acceptability and inferential judgments on ASL paradigms are made available in the Supplementary Materials.

\section{Elicitation methods and transcription conventions ${ }^{7}$}

\subsection{Elicitation methods}

The consultant (and co-author) is a Deaf, native signer of ASL (of Deaf, signing parents). ${ }^{8}$ Data were elicited using the 'playback method', with repeated quantitative acceptability judgments $(1-7$, with $7=$ best) and repeated inferential judgments (on separate days) on videos involving minimal pairs (see e.g. Schlenker et al. 2013, Schlenker 2014 for a description of the method). In a nutshell, the playback method involves two steps. First, the consultant signs sentences of interest on a video, as part of a paradigm (e.g. often with 2 to 6 sentences) of 'minimal pairs'. Second, the consultant watches the video, provides quantitative acceptability ratings, and (when relevant) inferential judgments, enters them in a computer, and redundantly signs all judgments on a video (for transparency, the raw written data are made available in the Supplementary Materials). The second step can be repeated on other days, sometimes with a considerable time delay. This method has the advantage of allowing for the precise

\footnotetext{
${ }^{7}$ This section borrows from Schlenker to appear a.

${ }^{8}$ We normally use the term 'consultant' to refer to data obtained in elicitation sessions (and thus prior to writing the article); if we report on 'Lamberton's' impressions, it is qua co-author, as he reflects on claims made in the article.
} 
assessment of minimal pairs (signed on the same video), in a quantitative, replicable way. Even when the judgments are obtained from just one consultant, the repetition of the task makes it possible to assess the stability of the judgments; and if necessary this method could be turned into an experimental one by assessing similar videos with other signers.

While this method has been used in quite a few publications in sign language semantics (see the survey in Schlenker 2017a), two remarks should be added. First, because we are interested in acceptability contrasts among sentences, the consultant is of course instructed to sign some sentences that are deviant or unacceptable; in fact, high ratings would be very hard to interpret if they did not contrast with low ratings for unacceptable sentences. Second, one might worry that the sentences are assessed by the very signer that produced them. It is clear that our results can only bear on the idiolect of a particular individual. But the work we conduct on this idiolect is arguably more rigorous than is common in non-experimental fieldwork, since (i) we distinguish the production phase from the judgment phase, and (ii) we provide multiple quantitative data points that make it possible to assess the stability (or lack thereof) of the judgments through time. This method should of course be assessed rigorously in the future, but this holds of other elicitation methods as well - and ours has the advantage of transparency (since the raw written data are made available in the Supplementary Materials).

For readability, only average judgments are given, as well as a summary of the relevant aspects of the inferential judgments (complete quantitative judgments are given when there is more than a 2point difference in the judgments obtained for a given sentence). Specialists are invited to consult the raw data when relevant. Note in particular that inferential judgments need not be straightforward to summarize, in which case the raw data may be particularly informative. In addition, the raw data include a description by the consultant himself of salient differences in the realization of signs. Notations such as $A S L, 34,1550 a, e, 5$ judgments indicate that the relevant sentences appeared in ASL video 34, 1550, that only sentences $a$ and $e$ (i.e. the first and the fifth) from that paradigm are transcribed, and that averages are computed on the basis of 5 judgments (if no letters followed 34, 1550, this would indicate that the entire paradigm was transcribed). When different inferential judgments were obtained on the same sentence, this is sometimes written with ratios, e.g. ' $3 / 5$ judgments' referring to ' 3 judgments out of $5^{\prime}$ (since a given judgment may mention several possible readings, judgments may sum to more than 5 in this case.)

\subsection{Transcription conventions}

In the following, sign language sentences are glossed in capital letters, as is standard. Translations were chosen to reflect, to the extent that this was possible, the inferential judgments that were given. ${ }^{9}$ When a translation appears after an entire paradigm, it holds for every sentence of the paradigm; otherwise a separate translation is provided for each example. When they matter, inferential judgments are explicitly represented and preceded by $=>$. The reader should consult the raw data for details, as summarizing inferential judgments is not always easy.

Expressions of the form WORD-i and WORD $D_{i}$ indicate that the relevant expression is associated with the locus (= position in signing space) $i$. A suffixed locus, as in WORD-i, indicates that the association is effected by modulating the sign in such a way that it points towards locus $i$; a subscripted locus, as in $W O R D_{i}$ or $[\ldots E X P R E S S I O N . . .]_{i}$, indicates that the relevant expression is signed in position $i$. Locus names are assigned from right to left from the signer's perspective; thus when loci $a, b, c$ are mentioned, $a$ appears on the signer's right, $c$ on the left, and $b$ somewhere in between. IX (for 'index') is a pointing sign towards a locus, while POSS is a possessive; they are glossed as $I X-i$ and POSS-i if they point towards (or 'index') locus $i$; the numbers 1 and 2 correspond to the position of the signer and addressee respectively. $I X-i$ is a standard way of realizing a pronoun corresponding to locus $i$, but the pointing sign $I X-i$ can also serve to establish rather than to retrieve a locus $i$. Agreement verbs include loci in their realization - for instance the verb $a-A S K-1$ starts out from the locus $a$ and targets the first person locus 1 ; it means that the third person individual denoted by $a$ asks something to the signer. When an expression indexes a default locus, it is usually written without a letter index (e.g. $I X$ rather than $I X-a$ ). $I X$-arc-i refers to a plural pronoun indexing locus $i$, as it involves an arc motion towards $i$ rather than a simple pointing sign. Some of the nouns we will use have two forms: an unmarked one, and one with a single repetition in the same location of signing space (hence a total of 2 iterations), typical of nouns. When this matters, the latter form is encoded by adding $n$ to the transcription. Thus TROPHY stands for the unmarked form, TROPHY- $n$ for the form with a single repetition.

\footnotetext{
${ }^{9}$ This is for instance the reason we include [possibly in a row] in the translation of (36)a: the specification 'in a row' or 'likely in a row' was provided by the consultant in some but not all judgment tasks.
} 
The suffix -rep is used for unpunctuated repetitions, and in such cases -rep3, -rep 4, -rep $5, \ldots$ indicate that there are $3,4,5, \ldots$ iterations. When relevant, we add a subscript indicating the shape of the repetition, e.g. rep $3_{\text {horizontal }}$ for a horizontal repetition (whether in a straight line or as a horizontal arc), -rep $3_{\text {triangle }}$ for a triangularshaped repetition. The suffix -cont is used for continuous repetitions, and subscripts may be used as well to indicate the shape of the movement, such as -cont horizontal $_{\text {or }}$-cont triangle. Punctuated repetitions of an expression WORD are encoded as [WORD WORD WORD] if they involve three iterations of that expression; [WORD WORD $W O R D]_{\text {horizontal }}$ and [WORD WORD WORD] $]_{\text {triangle }}$ provide information about the shape of the repetition.

Unless otherwise noted, non-manual expressions are not encoded in the transcriptions of signs or gestures.

\section{Iconic plurals and mass terms in ASL: initial properties}

In this section, we apply standard tests to establish a distinction between count and mass nouns in ASL, and briefly draw some connections with other findings on number in ASL (we refer the reader to Chierchia 2010 and Deal 2017 for recent semantic and typological studies of number across languages.)

\subsection{The count/mass distinction}

We will argue that $A-F E W$ is acceptable with count but not mass noun, whereas $A-L I T T L E$ has the opposite distribution. ${ }^{10}$ In particular, unpunctuated repetitions display properties otherwise associated with plurals. In addition, unpunctuated repetitions typically give rise to iconic inferences whereby the spatial arrangement of the repeated sign provides information about the arrangement of its denotation. Similar observations hold of mass terms that involve the continuous repetition of a sign.

Since sign form greatly matters in discussions of iconicity, it will be convenient to focus on a rather minimal pair, involving TOILET vs. PEE. Both expressions have the same manual component, involving a trembled $T$ of the manual alphabet, but for our consultant the mouthings are different: /to/ for TOILET, /pe/ for PEE.

Let us start with the paradigm in (6), in which different forms of TOILET co-occur with $A$ FEW (here 1_location means that the sign is repeated in a single location of signing space; 3 locations means that it is repeated in different locations, and area means that it is (continuously) repeated throughout an area).

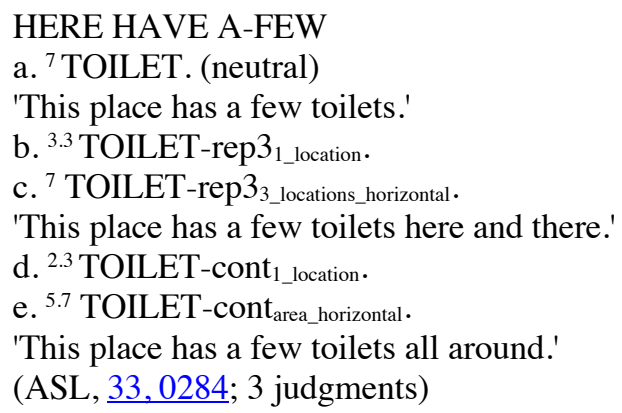

As can be seen, with $A-F E W$, a neutral form of TOILET can be used, as in (6)a, but an unpunctuated repetition can be used as well, as in (6)c, which involves 3 iterations in 3 different locations (spread out) of signing space. Iterating the sign in one and the same location, as in (6)b, yields deviance: pluralrelated repetition implies the use of different locations in space (other types of repetition in sign

${ }^{10}$ Koulidobrova 2018 does not report such contrasts in her ASL data; in particular, she gives an example in which either $M A N Y$ or $M U C H$ can be used with OIL:

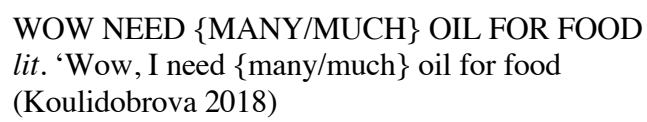

However Koulidobrova detects the presence of a count/mass contrast in the compatibility of different nominal expressions with numerical partitives. (We leave a comparison between our results and Koulidobrova's for future research.) 
language are not subject to this constraint). In (6)d,e, the sign is continually repeated, which is easy to realize with a trembled $T$ (one can just maintain the trembling for longer, and possibly over an entire area). Continuous repetition in a single location is sharply degraded, as seen in (6)d, whereas it is more acceptable if it covers an entire area, as in (6)e. The latter example is slightly degraded; there are two conceivable reasons: it could be that continuous repetition is not appropriate for count nouns, or that it is, but that it can only refer to very dense groups, which could contradict the use of $A-F E W$. Finally, weak iconic information is provided by repetition-based forms: (6)c is understood to mean that there are a few toilets spread out, in at least three locations. The somewhat degraded form in (6)e suggests that there are a few toilets grouped together and/or all over. (We come back below to examples in which iconic information is far more precise.)

It can immediately be checked in (7) that when $A-F E W$ is replaced with $A-L I T T L E$, the result is uniformly deviant - an unsurprising result if A-LITTLE selects for mass terms, as its English counterpart.

\author{
HERE HAVE A-LITTLE \\ a. ${ }^{3.7}$ TOILET. (neutral) \\ b. ${ }^{2}$ TOILET-rep $3_{1 \_ \text {location. }}$. \\ c. ${ }^{3.7}$ TOILET-rep33_locations_horizontal. \\ d. ${ }^{2}$ TOILET-cont ${ }_{1}$ Iocation. \\ e. ${ }^{3.7}$ TOILET-cont area_horizontal. $_{\text {. }}$. \\ (ASL, $\underline{33,0286 ;} 3$ judgments)
}

When TOILET is replaced with PEE, A-LITTLE becomes acceptable, as seen in (8)a. Unpunctuated repetition as in (8)c yields a reading on which urine is found in several areas, while continuous repetition as in (8)e indicates that there is urine all around. As before, repetition is deviant if it is realized in a single location, as in (8)b-d.

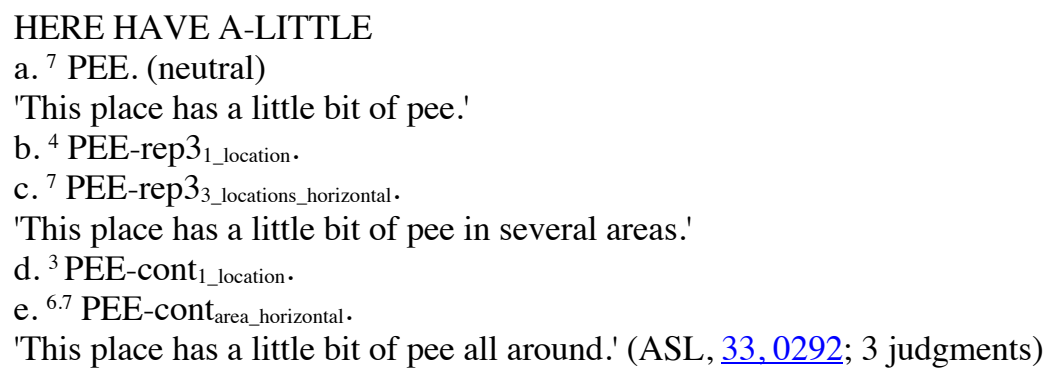

Turning to $P E E$ combined with $A-F E W$, we first find that the result is degraded when $P E E$ is in a neutral form, as in (9)a - which just suggests that $A-F E W$ selects for count terms, just like its English counterpart. Strikingly, (9)c shows that an unpunctuated repetition of $P E E$, using space, is acceptable in this context, but that it yields a reading on which there are a few areas of urine here and there. The repetition of the sign in discrete areas of signing space appears to be sufficient to 'countify' the mass term. ${ }^{11}$ By contrast, no such effect is found with continuous repetition over an area of space, as in (9)e: the result remains degraded, albeit with variations in the judgments.

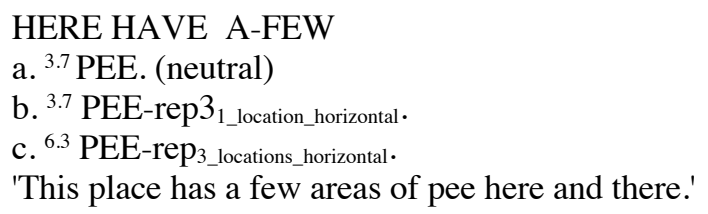

\footnotetext{
${ }^{11}$ We leave for future research a comparison between these 'countified' mass terms in ASL and related readings obtained with mass terms preceded by count quantifiers in some spoken languages, as described for instance in Lima 2014 (thanks to an anonymous reviewer for this reference). Lima shows that in Yudja (a Tupi language spoken in Brazil), "all numerals can be directly combined with mass nouns". She proposes that "Yudja is a language where all nouns can be construed as count nouns without coercion", and she argues that this is possible "because the grammar of the language allows its speakers to treat concrete portions of a kind as atoms".
} 


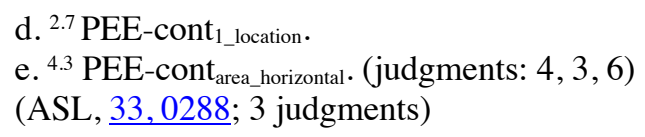

Several conclusions can already be drawn. First, plural readings can be obtained with neutral forms: in our data, repetition is not required in order to obtain a plural reading. Second, $A-F E W$ and $A$ LITTLE select for count and mass terms respectively, as their English counterparts do. Third, with respect to the nouns discussed above, if (plural-related) repetition is involved, it must use space: repetition in a single location is not acceptable. But this does not appear to be a general fact: Pfau and Steinbach 2006, cited above, discuss many cases of pluralization by way of repetition in one location; and for Lamberton, in the sentence GROW-UP IX-I WIN TROPHY-rep, meaning I won many trophies growing up, repetition in a single location is acceptable. Fourth, repetition-based forms with movement, whether continuous or not, provide iconic information about the arrangement of the denoted objects or stuff. Fifth, this iconic information may suffice to 'countify' a mass term so as to justify the use of $A$ $F E W$, yielding a reading on which areas of the relevant stuff are counted.

It is worth emphasizing that patterns involving a single repetition (i.e. a total of two iterations) in a single position is common in ASL to mark nominal (as opposed to verbal) category, without plural implications. The plural cases we will consider usually involve at least three iterations, but even when they involve just two, they sharply differ from repetition-qua-nominal marker in involving space.

\title{
3.2 Unrepeated vs. repeated forms
}

While our focus is on the interaction between plural repetitions and iconicity, we should briefly mention two questions that will interact with our analysis. First, are unrepeated nouns compatible with plural readings? Second, do repeated nouns range solely over strict pluralities (which contain at least two individuals), or can they also range over singletons?

\subsubsection{Can unrepeated forms range over non-singleton groups?}

Two arguments suggest that unrepeated nouns can range over pluralities. First, unrepeated nouns can co-occur with numerals, as illustrated in (10)-(11). By itself, this fact could be analyzed in a variety of ways. But several researchers have argued that numerals should be treated as modifiers (see Chierchia 2010, who in this respect follows Ionin and Matushansky 2006). If we assume that modification is intersective, 7 BOOK is true of those objects $\mathrm{x}$ such that $\mathrm{x}$ is in the extension of $B O O K$ and $\mathrm{x}$ is made of exactly seven objects. This requires that $B O O K$ in (10) should include strict pluralities in its extension, and similarly for TROPHY in (11).

\author{
${ }^{7}$ MUSEUM HAVE 7 BOOK. \\ 'The museum has 7 books.'

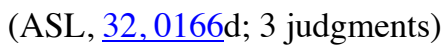 \\ (11) ${ }^{7}$ MUSEUM HAVE 7 TROPHY. \\ 'The museum has 7 trophies.'

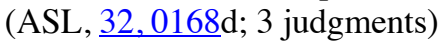

Second, earlier literature emphasized that unrepeated ASL nouns can have plural readings. Thus Petronio 1995 gives two possible translations for (12) (where in her transcription - preserved below $-t$ over MOVIE encodes topic marking). Similarly, she notes that depending on the context the first clause of (13) could mean: The student(s) is/are frustrated, and the teacher(s) is/are upset. Koulidobrova 2018 makes similar remarks. ${ }^{12}$

\footnotetext{
12 It should be added that both Petronio 1995 and Koulidobrova 2018 note that singularity entailments can be triggered by predicate classifiers, elements that provide information about the nature and position or movement of the denoted objects (they can typically be modulated to provide rich iconic information, as discussed in Emmorey and Herzig 2003). Thus Petronio 1995 contrasts the underspecification of (12) with the singularity entailment of (i), which (in her words) "contains a spatial verb with a singular classifier morpheme '1/1/' which
} 
(12)

MOVIE, WOMAN LOVE.

'The woman loved the movie.'

'The women loved the movie.'

(Petronio 1995)

(13) STUDENT FRUSTRATE, TEACHER UPSET.

(Petronio 1995)

We conclude that unrepeated ASL nouns can contain strict pluralities in their extension.

\subsubsection{Can repeated forms range over singletons?}

In English, it is now commonly assumed that plurals can range over singletons, and that the nonsingleton inferences triggered in some environments are due to pragmatic enrichment (e.g. Sauerland 2003, Spector 2007, Chierchia 2010). The main argument for this conclusion is that negative environments such as those in (14)a,b do not just exclude the presence of non-singleton groups of problems, but also of singleton problems. If plurals only ranged over strict pluralities, the readings obtained would be weaker: (14)a would deny that the homework contains at least two difficult problems, and (14)b would deny that any of the speaker's students solved at least two difficult problems. Similarly, the condition introduced by the if-clause in (14)c is arguably satisfied as soon as the homework contains at least one difficult problem, whereas a strict plural analysis would predict the condition to be satisfied only in case the homework contains at least two difficult problems.

(14) a. The homework doesn't contain difficult problems.

(Spector 2007)

b. None of my students has solved difficult problems.

(Spector 2007)

c. If the homework contains difficult problems, I'll give you $\$ 10$.

While to our knowledge a similar issue has not been investigated in detail for ASL repetitionbased nouns, some examples suggest that unrepeated nouns only range over non-singleton (and possibly larger) groups. Thus Koulidobrova 2018 considers the yes-no question in (15)A, with a noun TREE repeated with sideward movement (which she transcribes as $+>+>+>$ ). The answer in $B$ is deviant, whereas that in $\mathrm{B}^{\prime}$ is acceptable; this suggests that repeated TREE comes with a non-singleton entailment.

(15)

A: HAVE TREE+>+>+> HERE

'Do you have trees?'/ 'Are there trees here?'

B: \#YES, HAVE ONE PINE

'Yes, we have one pine'

B': NO, ONLY ONE

'No, only one'

(Koulidobrova 2018)

Similarly, we have examples such as (16), where despite the small number of iterations (just two), the if-clause is satisfied only in case at least two or three books are present $((26) \mathrm{b}$ and (28)c below make the same point).

corresponds to a singular person" ( $a$ is the locus introduced by STORE and targeted by the - moving - person classifier).

(i)

aSTORE, WOMAN CL: /1/' 'go to' a.

'The woman went to the store.'

(Petronio 1995) 
(16) Context: The speaker will be renting the addressee's apartment.

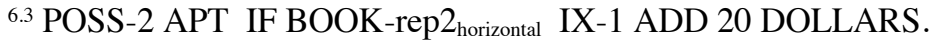

'If your apartment has at least 2 or 3 books in a row, I will add \$20.'

(ASL, 32, 0028b; 3 judgments; see (27) below for further details)

Pending further investigation, it would seem that (some) repetition-based plurals are constrained to range over non-singleton groups. In fact, they might be constrained to range over larger groups, since the conditions we will see below (especially in (26)b and (28)c) make reference to at least three objects of the relevant kind. But as we will see in Section 9.2, it is not clear whether these nonsingleton requirements are due to the logical or to the iconic component of repetition-based plurals.

\section{Unpunctuated repetitions as plurals: grammatical properties}

We turn to a more detailed study of unpunctuated repetitions. We discuss their grammatical properties in this section, and explore their iconic properties in the next.

\subsection{Co-occurrence with numerals}

In (17) and (18), three forms of the nouns BOOK and TROPHY are contrasted: a basic one, without repetition, in (17)a,d and (18)a,d; one with a single repetition typical of nouns, in (17)b,e and (18)b,e (as noted above, the two iterations are signed in the same position); and one with several repetitions typical of plurals, in (17)c,f and (18)c,f. As far as we can tell, the distinction between the nominal form and the neutral form disappears in the plural, which is why we investigate a single type of plural form here. ${ }^{13}$

(17) MUSEUM HAVE

a. ${ }^{7} 1$ BOOK.

'The museum has 1 book.'

b. ${ }^{5.7} 1$ BOOK-n.

'The museum has 1 book.'

c. ${ }^{4.7} 1$ BOOK-rep $3_{\text {horizontal }}{ }^{14}$

d. ${ }^{7} 7$ BOOK.

'The museum has 7 books.'

e. ${ }^{6.7} 7$ BOOK-n.

'The museum has 7 books.'

f. ${ }^{6.7} 7$ BOOK-rep3 $3_{\text {horizontal }}$.

'The museum has 7 books arranged in a row.'

(ASL, 32, 0166; 3 judgments)

(18) MUSEUM HAVE

a. ${ }^{7} 1$ TROPHY.

'The museum has 1 trophy.'

b. ${ }^{7} 1$ TROPHY-n.

'The museum has 1 trophy.'

c. ${ }^{5} 1$ TROPHY-rep3 $3_{\text {horizontal }}$

d. ${ }^{7} 7$ TROPHY.

'The museum has 7 trophies.'

e. ${ }^{7} 7$ TROPHY-n.

'The museum has 7 trophies.'

f. ${ }^{7} 7$ TROPHY-rep $3_{\text {horizontal }}$.

\footnotetext{
${ }^{13}$ Lamberton does not perceive a meaning or a grammatical distinction between $B O O K$ with and without nominal repetition. (While $B O O K$ without nominal repetition could in principle lead to confusions with the verbal form $O P E N-B O O K$, the risk is limited because in this case the verb is quite a bit less common than the noun. TROPHY without nominal repetition shouldn't yield an ambiguity with the verb $A W A R D$, as the latter has an agreement requirement.)

${ }^{14}$ In the last session, a rating of 2 or 4 was given depending on the scenario: 2 if the museum has a single book, 4 if it has a single row of books (see the Supplementary Materials for details). We computed the average taking into account the highest score, since the context allows for the scenario in which the sentence has that score.
} 
'The museum has 7 trophies arranged in a row.'

(ASL, $\underline{32,0168 ;} 3$ judgments)

Two observations should be made. First, as noted in Section 3.2.1, a numeral such as 7 can definitely be followed by a non-plural form, as in (17)d,e and (18)d,e (these correspond to what Pfau and Steinbach 2006 analyze in DGS [German Sign Language] as zero-marked forms). Second, the unpunctuated repetitions come with an iconic interpretation, according to which the books and trophies are standing upright and appear in a row - a remark we already made in connection with our initial data in Section 3.1.

\subsection{Cumulative readings}

Turning to the semantic side, unpunctuated repetitions can give rise to the same kind of cumulative readings as standard plurals. Thus both the unmarked nouns in (19)a and the unpunctuated repetitions in (19)b allow for (but of course do not force) a cumulative reading, according to which 200 hens collectively laid 300 eggs. And as was observed for spoken language (Zweig 2006), the cumulative reading becomes unavailable when MOST replaces the subject determiner, as in (19)c,d, which results in a distributive inference to the effect that some hens individually laid 300 eggs. The facts are similar in the English paradigm in (20).

(19) a. ${ }^{6.3} 200$ HEN FINISH LAY 300 EGG.

b. ${ }^{5.7} 200$ HEN-rep3 $3_{\text {horizontal }}$ FINISH LAY 300 EGG-rep3 ${ }_{\text {horizontal }}$.

'200 hens laid 300 eggs.' (= cumulative reading available)

c. ${ }^{7}$ HEN MOST FINISH LAY 300 EGG.

d. ${ }^{6.3}$ HEN-rep3 ${ }_{\text {horizontal }}$ MOST IX-arc FINISH LAY 300 EGG-rep3 $3_{\text {horizontal. }}$.

'Most hens have laid 300 eggs.' (= cumulative reading unavailable)

(ASL, 32, 0190, 3 judgments)

(20) a. 200 hens lay 300 eggs.

\#> some hens each lay 300 eggs

Possible reading: a total of 200 hens lay eggs, and a total of 300 eggs were laid by these hens.

b. Most hens lay 300 eggs.

=> some hens lay 300 eggs.

Reading: for most hens $\mathrm{x}, \mathrm{x}$ lay $300 \mathrm{eggs}$

\subsection{Dependent plurals?}

Despite their optionality and iconic semantics, repetition-based plurals display some non-trivial properties of standard plurals. Most strikingly, they give rise to readings that are reminiscent of 'dependent plural' uses, whereby a plural that is dependent on another plural yields what seems to be a singular reading (see Zweig 2006 for spoken language data, and further references). Concretely: the sentences in (21) certainly do not entail that any book has several front covers.

(21) a. All books have nice front covers.

b. Most books have nice front covers.

The same data can be found with repetition-based plurals in ASL. ${ }^{15}$ This can be seen in (22)c, which would be very odd if the second plural were interpreted in the scope of the first plural, as this would imply that some books have several front covers.

a. ${ }^{7}$ ALL BOOK HAVE NICE FRONT COVER.

b. ${ }^{6.7}$ ALL BOOK-rep $3_{\text {horizontal }}{ }^{16}$ HAVE NICE FRONT COVER.

\footnotetext{
${ }^{15}$ The repetition-based forms seem to come with an implication that we are talking about a particular set of books; this is arguably a consequence of the iconic semantics which is described below.

${ }^{16}$ For phonological reasons, BOOK can't take on a smooth continuous form like TOILET, hence the choice between the -rep vs. -cont transcription is less clear in this case.
} 
c. ${ }^{6.7}$ ALL BOOK-rep3 $3_{\text {horizontal }}$ HAVE NICE FRONT COVER-rep3 $3_{\text {horizontal }}$.

'All books have nice front covers.' (ASL, 32,0178; 3 judgments)

The same observation extends to the paradigm in (23), where we took the precaution of asking the consultant in some judgment tasks whether there was an implication that some books have several front covers (there wasn't).

(23) a. ${ }^{7}$ MOST BOOK HAVE NICE FRONT COVER.

b. ${ }^{6.5}$ MOST BOOK-rep3 $3_{\text {horizontal }}$ HAVE NICE FRONT COVER.

c. ${ }^{6}$ MOST BOOK-rep3 $3_{\text {horizontal }}$ HAVE NICE FRONT COVER-rep3 $3_{\text {horizontal }}$.

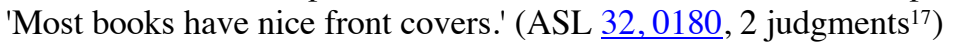

$=>$ no implication that any book has several front covers

It is outside the scope of this piece to explain why dependent plurals are possible in the first place, but the environments we chose are particularly informative because all and most were argued by Zweig 2006 to allow for dependent plurals but not to give rise to cumulative readings, as illustrated in (24)-(25) (where $\neq>$ indicates that the cumulative reading is missing). The latter fact (no cumulative readings) was established in (19)c,d for MOST in ASL. This suggests that dependent readings are not reducible to cumulative readings, both in English and in ASL.

(24) a. Most students read thirty papers.

F> Most students read at least 1 paper, and a total of 30 papers were read overall.

b. Most students read papers.

F> Most students read at least 1 paper, and more than 1 paper was read overall (Zweig 2006)

(25) a. All the students read thirty papers.

\#> All the students read at least 1 paper and a total of 30 papers were read overall.

b. All the students read papers.

\#> All the students read at least 1 paper and more than 1 paper was read overall (Zweig 2006)

It is an open question whether the dependency we find in (22)-(23) is fully identical to that in (21). There are reasons to doubt it, in particular because there are mass equivalents of dependent plurals, which provide iconic information about the nature of the relation between two substances (we come back to this point with mass terms in Section 8.3; we have not specifically tested the contribution of iconic information in relation to dependent repetition-based plurals - a point we leave for future research).

Be that as it may, these observations only scratch the surface of the grammatical and semantic behavior of ASL unpunctuated repetitions. But they suggest that they share important properties of standard plurals, and thus that their iconic properties should be viewed as an enrichment of standard plural semantics rather than as an entirely different phenomenon.

\section{Unpunctuated and punctuated repetitions: iconic properties}

\subsection{Main properties}

We now consider in greater detail the iconic properties of unpunctuated repetitions as well as punctuated repetitions, which in this respect display the same general behavior. The main difference between them pertains to the threshold conditions they introduce: punctuated repetitions impose precise thresholds (e.g. 'at least three books' if there are three iterations), whereas unpunctuated repetitions usually impose vague conditions (e.g. 'at least three or four books'). Their vagueness can be diagnosed in two ways. First, vagueness can give rise to explicit uncertainty on the consultant's part, who may in a given

\footnotetext{
${ }^{17}$ We thank an anonymous referee for urging us to add this paradigm. Judgments had been collected in only 2 sessions before the article was completed, with inferential judgments in only one of them. We re-tested this paradigm later, with the same overall results: 7 for all three examples, no inference that a book has several front covers. (We also tested on 3 occasions a paradigm (ASL, 32, 0186) that included variations of (23)c with MOST $B O O K$-rep $3_{\text {horizontal }}$ replaced by BOOK-rep $3_{\text {horizontal }}$ MOST or by BOOK-rep $3_{\text {horizontal }}$ MOST IX-arc. Their acceptability was even higher and the inferential judgments were the same.)
} 
inferential judgment state in one way or another that it is unclear whether the sentence counts as true if there are 3 books, for instance; we describe this case below as 'explicit uncertainty'. Second, vagueness can be diagnosed across judgments, if the consultant mentions different thresholds in different iterations of the task.

Importantly, the existence of a precise threshold for punctuated repetitions does not entail that these get 'exactly' readings. In fact, most punctuated repetitions trigger inferences to the effect that there are, say, at least three objects satisfying the relevant condition: this is an 'at least' reading with a precise threshold. (Our translations are intended to summarize the inferential judgments, including in cases of uncertainty, sometimes marked with question marks; but the Supplementary Materials should be consulted for details.)

\subsubsection{Number and speed of the repetitions}

The paradigm in (26) contrasts a punctuated repetition of the neutral form of $B O O K$ (without the nominal doubling discussed above), with several unpunctuated repetitions. For the latter, we varied the number of repetitions (three vs. four) and their speed (slow vs. fast).

(26) Context: The speaker will be renting the addressee's apartment.

POSS-2 APT IF HAVE

'If your apartment has

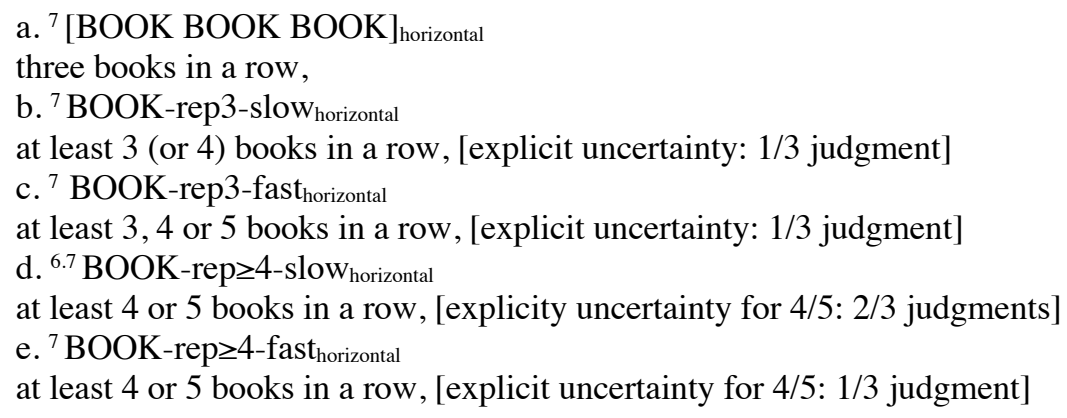

IX-1 ADD 20 DOLLARS.

I'll add \$20.' (ASL, $\underline{32,0046 ;} 3$ judgments)

Note: On 2/3 judgments, the 'cut-off' for e. was judged to be likely higher than for $\mathrm{d}$.

A separate paradigm (without $H A V E$ ) also provided some information about more extreme cases with two and five repetitions, but without varying the speed (for simplicity, we do not report all the conditions tested in this paradigm).

(27) Context: The speaker will be renting the addressee's apartment.

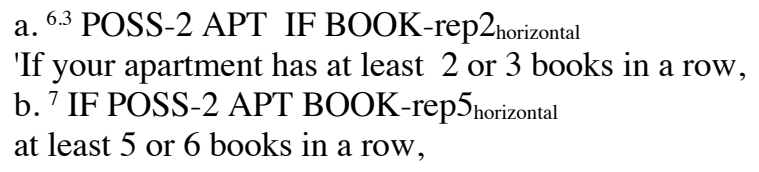

IX-1 ADD 20 DOLLARS.

I will add \$20.' (ASL, 32, 0028b,e; 3 judgments)

In all conditions, the iterations were performed in a horizontal row, and in (26) all gave rise to iconic readings on which the books should be horizontally aligned for the condition to hold (in (27), iconic inferences were not discussed in the judgments; we come back to iconic conditions in the next paragraph). But as was stated at the outset, there are subtle differences in the numerical conditions. Punctuated iterations give rise to precise readings on which the condition specifies that there should be at least three aligned books (we will refine this initial generalization in Section 7). Unpunctuated repetitions usually give rise to vague readings on which the threshold can be explicitly seen as uncertain 
and/or can give rise to inconsistent readings across trials. Furthermore, larger numbers of repetitions and (to some extent) greater speed are associated with larger quantities. ${ }^{18}$

\title{
5.1.2 Shape
}

To bring into sharper focus the import of shape, we turn to the paradigm in (28), which contrasts a horizontal and a triangular arrangement of the repetitions, both punctuated and unpunctuated; pictures have been added to help the reader visualize the two shapes in key conditions, and a lightly anonymized video has been made available as well. The horizontal version involves repetition of the sign in a leftto-right row in front of the signer, with the shape: ... ; the triangular version involves a vertical triangle signed from left to right, with an iteration on the two bases on the left and right as well as on the tip above in the middle, with the shape: $\therefore$ (in the case of at least 4 iterations, at least one must be elsewhere on the triangle; this is relevant for (28)f, although in this case the shape is a bit indistinct). There are clear truth-conditional differences among these sentences, and the iconic contribution of the repeated noun is interpreted within the scope of the conditional, which suggests that it is at-issue. (We checked in the last judgment task that these sentences do not trigger any inference to the effect that if there are trophies, they should be arranged in a particular way; this was to ascertain that there is no 'projection' of the inference pertaining to the arrangement of the relevant objects. ${ }^{19}$ )

(28) Context: The speaker will be renting the addressee's apartment; he knows it contains trophies, but he hasn't seen them.

POSS-2 APT IF HAVE

'If your apartment has , IX-1 ADD 20 DOLLARS.

a. ${ }^{7}[\text { TROPHY TROPHY TROPHY }]_{\text {horizontal }}$

$=$ three trophies forming a line

$\Rightarrow>$ if there at least three trophies in a horizontal line, $\$ 20$ will be added. Precise condition about numbers: no hesitation for the 'exactly 3 ' condition

b. ${ }^{7}[\text { TROPHY TROPHY TROPHY }]_{\text {triangle }}$

$=$ three trophies forming a line

$\Rightarrow>$ if there at least three trophies forming a triangle, $\$ 20$ will be added. Precise condition about numbers: no hesitation for the 'exactly 3 ' condition

c. ${ }^{7}$ TROPHY-rep3 $3_{\text {horizontal }}$

\begin{abstract}
${ }^{18}$ Thanks to Jeremy Kuhn (p.c.) for suggesting that speed could matter independently from number of repetitions. One further remark is in order. Contextual knowledge seems to play a role in the interpretation of the quantities denoted by plurals. As an example, consider the minimally different examples in (i)-(ii), which both involve unpunctuated repetitions, but with $B O O K$ in (i) and TROPHY in (ii). The consultant noted in one judgment task that the office-related context led one to understand that there were at least 10 books, whereas one could understand that there were at least 4 trophies. This is presumably because one expects that more books than trophies would be found in an office, and thus that the (vague) thresholds imposed by unpunctuated repetitions may differ in the two cases.
\end{abstract}

POSS-2 OFFICE HAVE BOOK-rep3 $3_{\text {horizontal. }}$.

'Your office has (at least 10+) books.' (ASL, 34, 2190)

POSS-2 OFFICE HAVE TROPHY-rep3 $3_{\text {horizontal }}$.

'Your office has (at least 4+) trophies.' (ASL, 34, 2192)

${ }^{19}$ This test matters because if the iconic conditions behaved like co-speech gestures as analyzed in Schlenker to appear c, d, one would expect an inference to the effect that if there are trophies, they are arranged in a linear/triangular fashion. 


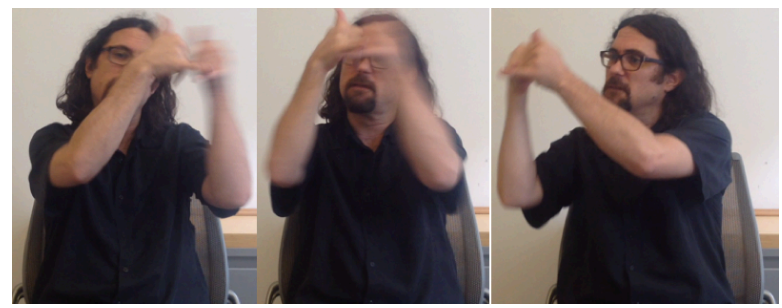

$=$ several trophies forming a line

$\Rightarrow>$ if there at least three or four trophies in a horizontal line, $\$ 20$ will be added. Vague condition about numbers: explicit uncertainty for the 'exactly 3 ' condition ( $2 / 4$ judgments)

d. ${ }^{6.7}$ TROPHY-rep $3_{\text {triangle }}$

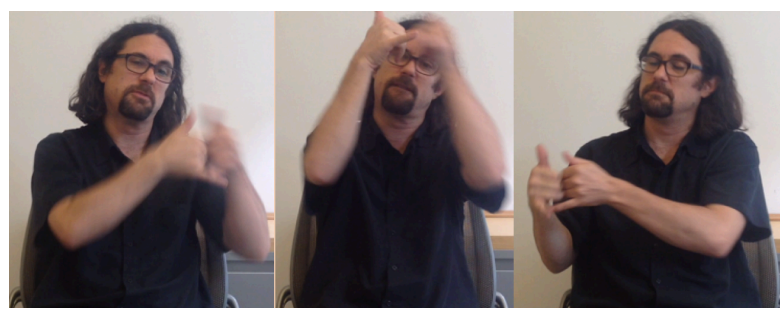

$=$ several trophies forming a triangle

$\Rightarrow>$ if there are at least 3 trophies forming a triangle, $\$ 20$ will be added. Explicit uncertainty if there is a large number of trophies in a row (4/4 judgments)

e. ${ }^{6.7}$ TROPHY-rep $\geq 4_{\text {horizontal }}$

$=$ quite a few trophies forming a line

$\Rightarrow>$ if there at least three or four or five trophies in a horizontal line, $\$ 20$ will be added. Vague condition about numbers: explicit uncertainty for the 'exactly 3' (2/4 judgments) and 'exactly 4' (1/4 judgments) conditions

\section{f. ${ }^{6.5}$ TROPHY-rep $\geq 4$ triangle $=$ quite a few trophies forming a triangle}

$\Rightarrow>$ if there are at least three or four or five trophies forming a triangle, $\$ 20$ will be added. Vague condition about numbers: explicity uncertainty for the 'exactly 3 ' (2/2 judgments) and 'exactly 4' (1/4 judgment) conditions. Explicit uncertainty if there is a large number of trophies in a row (3/4 judgments).

(ASL, $\underline{32,0096}, 4$ judgments. A lightly anonymized video can be found online: https://youtu.be/muSEPdasgew)

Two further remarks should be added. First, as was the case in the paradigm in (26), the number of repetitions is interpreted, with larger numbers of repetitions associated with larger groups, and this condition too appears to be at-issue. Second, as was already the case in (26), there is a subtle distinction between punctuated and unpunctuated repetitions: the former yield precise thresholds, in the sense that it should be possible to find objects that can serve as the denotation of each punctuated iteration (with no requirement that this be exhaustive, as this reading allows for the presence of further objects that satisfy the condition: this is a precise 'at least' reading). With unpunctuated repetitions, threshold conditions pertaining to the number of objects are vague, which can be diagnosed by way of explicit judgments of uncertainty about quantitative thresholds, and by inconsistencies across judgments. In addition, there seems to be some vagueness about the shape condition with unpunctuated repetitions, as a large number of objects in a row may to some extent satisfy the triangular condition (furthermore, the shape in (28)d,f is a bit indistinct $)^{20}$. In fact, in Lamberton's judgment, there is some amount of vagueness in both shape conditions: to some extent, objects not in a row could satisfy the row condition, and objects in a row could satisfy the triangular condition, although the inferential questions show clear contrasts. (The reader is referred to the Supplementary Materials for the full judgments, which are hard to summarize.)

\footnotetext{
${ }^{20}$ As Lamberton notes, the triangular shape in (28)d could be an arrow rather than a triangle; and the corresponding shape in (28)f is a bit smoother, with the result that some could see an arch instead of a triangle.
} 
To conclude, in some cases at least, punctuated and unpunctuated repetitions come with iconic conditions as part of their at-issue contribution. The number and speed of repetitions appear to be interpreted, with larger numbers and greater speed corresponding to larger quantities. Unpunctuated repetitions differ from punctuated ones in that the former but not the latter come with vague conditions on numerical thresholds, which can be diagnosed by way of explicit judgments of uncertainty or by inconsistencies across judgments for one and the same sentence.

\subsection{Initial analysis}

In view of the role of iconic conditions in the semantics of unpunctuated repetitions, it is clear that interpretive rules must make provisions for requirements of the form: the denotation of expression $E$ must resemble E along certain dimensions. As announced, we will not be concerned with the details of these iconic conditions, but only with the way in which they interact with logical conditions. ${ }^{21}$ We treat unpunctuated repetitions as plurals with iconic conditions. We only sketch possible accounts of punctuated repetitions, as their semantics is revisited in Section 7.

\subsubsection{Unpunctuated repetitions}

We start from a very simple analysis of plurals in English. We follow the literature (most recently, Nouwen 2015) in taking a plural $N P$ such as books to denote the set of sums of individual books, as stated in (29). (29)a defines an operator, *, which takes a set of atomic objects (for instance of individual books, $\left\{b_{1}, b_{2}\right\}$ ) and returns the set of mereological sums of these objects (in our example, $\left\{b_{1}, b_{2}\right.$, $\left.\mathrm{b}_{1}+\mathrm{b}_{2}\right\}$. If book denotes the set $\left\{\mathrm{b}_{1}, \mathrm{~b}_{2}\right\}$, books will denote the set $\left\{\mathrm{b}_{1}, \mathrm{~b}_{2}, \mathrm{~b}_{1}+\mathrm{b}_{2}\right\}$, a stated in $(29) \mathrm{b}^{22}$ Adding existential quantification, we obtain the analysis in (30), illustrated on the example of some books.

Notation: As is standard, if $\mathrm{s}$ is an assignment function, $\mathrm{s}[\mathrm{X} \rightarrow \mathrm{d}]$ is the assignment function which is identical to $\mathrm{s}$, with the possible difference that it assigns to the variable $X$ the value $\mathrm{d}$.

(29) English: plural NPs (e.g. Nouwen 2015)

a. Link's sum closure operator: if $\mathrm{X}$ is a set, ${ }^{*} \mathrm{X}$ is the smallest set such that:

(i) $\mathrm{X} \subseteq * \mathrm{X}$

(ii) for all $\mathrm{x}, \mathrm{x}^{\prime}$, if $\mathrm{x} \in \mathrm{X}$ and $\mathrm{x}^{\prime} \in \mathrm{X}$, then $\mathrm{x}+\mathrm{x}^{\prime} \in \mathrm{X}$, where $\mathrm{x}+\mathrm{x}^{\prime}$ is the mereological sum of $\mathrm{x}$ and $\mathrm{x}^{\prime}$

Terminology: we will call members of $* \mathrm{X}$ groups.

b. $[$ books $]]=*[$ book $]]$

${ }^{21}$ Further research should explore how iconic conditions themselves should be precisely stated; for analyses in which iconic conditions require denotations to preserve some formal geometric properties of expressions, see Greenberg 2013, Schlenker et al. 2013, Kuhn 2015, Kuhn and Aristodemo 2017, and Schlenker to appear b, d.

${ }^{22}$ One often takes books to also be true of individual books, for instance to guarantee that I have no books is falsified in case I have a single book. See for instance Spector 2007 for a far more sophisticated discussion. 
(30) English: plural quantification

If $\mathrm{c}$ is a context of evaluation and $\mathrm{s}$ an assignment function, and if $P$ is propositional expression (typically one with with a free variable $\left.X^{23}\right)$, [[[some books $\left.]_{\mathrm{X}} \mathrm{P}\right]^{\mathrm{c}, \mathrm{s}}=$ true

iff for some object $\mathrm{d}$, [[books $](\mathrm{d})=$ true $^{24}$ and $[[\mathrm{P}]]^{\mathrm{c}, \mathrm{s}[\mathrm{X} \rightarrow \mathrm{d}]}=$ true, iff for some object $\mathrm{d}, \mathrm{d} \in *[[$ book] $]$ and

$[\mathrm{P}]^{\mathrm{c}, \mathrm{s}[\mathrm{X} \rightarrow \mathrm{d}]}=$ true,

iff for some object $\mathrm{d}, \mathrm{d}$ is a group of books and $[[\mathrm{P}]]^{\mathrm{c}, \mathrm{s}[\mathrm{X} \rightarrow \mathrm{d}]}=$ true.

To account for iconic conditions triggered by unpunctuated repetitions in ASL, we propose to add to (30) the boldfaced clause shown in (31). For ease of comparison with English, we initially assume that $B O O K$ is just true of atomic books, but we will revisit this simplification in Section 9. Our rule is relativized to the context of evaluation because one and the same iconic sign can probably impose different conditions depending on the context, and the context might also specify how fine-grained the representation is (a point to which we return below). One might take this boldfaced clause to be optional if one thinks that there are iconically 'neutral' unpunctuated repetitions, which do not provide iconic information about their denotations. But one could also handle this case by allowing the relation 'iconically represents' to be trivialized in some cases (we return to this point in Section 6.4).

(31) ASL unpunctuated repetitions (to be revised)

If $\mathrm{c}$ is a context of evaluation and s an assignment function, and if $P$ is a propositional expression (typically one with a variable $X)^{25}$,

$\left[\left[\mathrm{BOOK}-\mathrm{rep}_{\mathrm{X}} \mathrm{P}\right]\right]^{\mathrm{c}, \mathrm{s}}=$ true

iff for some object $\mathrm{d}$, $*\left[[\mathrm{BOOK}](\mathrm{d})=\right.$ true and $\boldsymbol{B O O K}-\boldsymbol{r e p} \boldsymbol{p}_{X}$ iconically represents $\mathrm{d}$ given $\mathrm{c}$ and $[[\mathrm{P}]]^{\mathrm{c}, \mathrm{s}[\mathrm{X} \rightarrow \mathrm{d}]}=$ true,

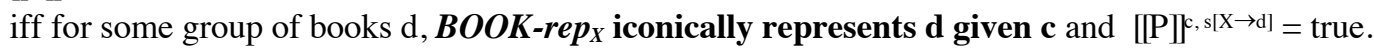

We take not just the shape of $B O O K$-rep but also the number and the speed of the repetitions to play a role in iconic representations, and thus we do not specify separate conditions pertaining to the number of objects within the group d, as these would arguably be redundant. ${ }^{26}$ Of course a theory of 'iconic representation' will need to be plugged in this definition to obtain a complete specification of the truth conditions. But it is worth pointing out that there are precedents for treating sign language expressions as being simultaneously linguistic and iconic: precisely this view was proposed for loci in Schlenker et al. 2013, which argued that in some cases these are both logical variables and simplified pictorial representations of what they denote (we come back to a related point in Section 6.3). Closer to our topic, our analysis follows the spirit of Kuhn and Aristodemo's treatment of LSF repetition-based pluractionals. Specifically, as was noted in Section 1.3, Kuhn and Aristodemo 2017 took these pluractionals to come with a logical and an iconic condition, and crucially the latter was treated as being at-issue - which is precisely what we are saying about the iconic contribution of repetition-based plurals.

To illustrate the analysis, consider the simplified example in (32). For simplicity, we assume that the indefinite $B O O K$-rep undergoes 'Quantifier Raising' and binds a trace in object position, leading to the Logical Form in (32)b, which is then interpreted as in (32)c by applying the interpretive rule in (31). (We assume a standard system in which POSS-2 APARTMENT and the object trace are of type e, while HAVE is of type $<\mathrm{e},\langle\mathrm{e}, \mathrm{t}\rangle\rangle$.)

(32) a. POSS-2 APARTMENT HAVE BOOK-rep.

b. BOOK-rep $\mathrm{p}_{\mathrm{x}}\left[\right.$ POSS-2 APARTMENT] HAVE $\left.t_{\mathrm{x}}\right]$

${ }^{23}$ The rule can be applied even in the absence of a variable $X$ in the propositional expression $P$; if so, the quantifier [some books] $X$ will bind nothing. But on standard assumptions (e.g. Heim and Kratzer 1998), the syntax will not allow such a case to arise (e.g. on the assumption that [some books] $]_{X}$ originates in an argument position and gets to a scope position by 'Quantifier Raising', leaving behind a coindexed trace).

${ }^{24}$ Note that for the general case we could replace the condition $[\mathrm{NPs}](\mathrm{d})=1(\mathrm{e} . \mathrm{g}$. [[books $\left.](\mathrm{d})=1\right)$ with $\left.[\mathrm{NPs}]\right]^{\mathrm{c}}$, ${ }_{s[\mathrm{X} \rightarrow \mathrm{d}]}(\mathrm{d})=1$, as a complex NP might contain context- and assignment-dependent elements. This is immaterial for the present discussion.

${ }^{25}$ Since the condition on the presence of $X$ in the propositional expression $P$ is not a necessary part of the definition, it will not be further repeated in semantic rules below.

${ }^{26}$ It is because of these quantitative conditions that unpunctuated repetitions give rise in our examples to inferences (and translations) that do not just involve books, say, but books together with certain numerical requirements. 
c. $[[(b)]]^{c, s}=$ true

iff for some group of books d, BOOK-rep $\boldsymbol{B}_{X}$ iconically represents d given $\mathbf{c}$ and [IPOSS-2 APARTMENT HAVE $\left.\left.t_{x}\right]\right]^{c, s[X \rightarrow d]}=$ true,

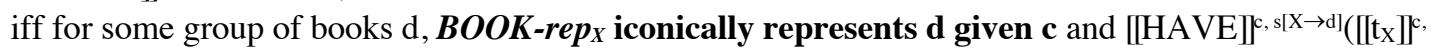
$\left.{ }_{s[X \rightarrow \mathrm{d}]}\right)\left(\left[[\mathrm{POSS}-2 \text { APARTMENT }]^{\mathrm{c}, s[X \rightarrow \mathrm{d}]}\right)=\right.$ true,

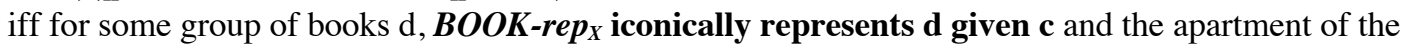
addressee of $\mathrm{c}$ has $\mathrm{d}$.

\subsubsection{Punctuated repetitions (to be revisited)}

Having seen how iconic conditions constrain the (plural) semantics of unpunctuated repetitions, we should ask how they interact with punctuated repetitions. But this presupposes an answer to a more elementary question: what kind of logical contribution is made by punctuated repetitions? In view of the data discussed so far, an initial hypothesis could be that each of the punctuated iterations introduces a separate indefinite, with covert conjunctions between them, or alternatively with a conjunctive meaning for a list of indefinites. Thus a simplified sentence such as POSS-2 APARTMENT HAVE [BOOK BOOK BOOK $]_{\text {horizontal }}$ could be taken to mean something like: Your apartment has a book and another book and a third book, with the iconic condition specifying that the books are arranged in a row. This analytical direction is motivated by three observations: first, formally these iterations have the form of repeated NPs; second, in the examples discussed, punctuated repetitions seem to come with threshold conditions on which each iteration stands for one object (hence a precise threshold when we obtain existential readings); third, in simple examples, each iteration can serve as an antecedent for further anaphoric uptake, as we will see in Section 6.

We do not seek to further implement this analysis of punctuated repetitions as conjoined indefinites because we will see in Section 7 that it is incorrect. The heart of the matter is that an expression such as 10 [TROPHY TROPHY TROPHY] is acceptable (and makes reference to ten trophies), which makes little sense if we are dealing with a conjunction of three singular indefinites. We will argue instead that punctuated repetitions should be seen as plurals with iconic conditions that entail that, as a default, it should be possible to find one separate denotation for each iteration.

\section{Unpunctuated and punctuated repetitions: interaction with anaphora}

Our analysis of unpunctuated repetitions as plurals predicts that these should introduce a plural discourse referent, and no singular discourse referents. By contrast, if punctuated repetitions are analyzed as conjoined indefinites, each iteration of the noun should introduce its own singular discourse referent. If we combine this observation with a dynamic view of discourse referents (as argued for sign language in Schlenker 2011b), we expect that each such discourse referent could support separate instances of singular anaphora in discourse.

The prediction pertaining to punctuated repetitions is generally borne out (with possible exceptions that are briefly discussed at the end of Section 7.2). But we will see in this section that the prediction about unpunctuated repetitions is incorrect: these can, under restricted conditions, introduce singular discourse referents, and we will argue that a more detailed understanding of iconic semantics might explain why. (Since we will in the end refute the analysis of punctuated repetitions as conjunctions of singular indefinites, we will also need to provide another account of the fact that each punctuated iteration introduces a separate discourse referent; we will do so by extending to punctuated repetitions the iconic analysis developed for unpunctuated repetitions).

\subsection{Singular discourse referents introduced by iconic plurals: the Edge Effect}

In the paradigm in (33), each iteration of $B O O K$ in the punctuated repetition can serve as an antecedent for the possessive pronoun POSS (we systematically use possessives as anaphoric pronouns because regular pronouns realized as pointing signs might have irrelevant readings, including locative ones). This is expected on the account of punctuated repetitions as conjoined indefinites, as we briefly suggested in Section 5.2.2. (although as mentioned this account will turn out to be incorrect). What isn't expected, on the other hand, is that the right and left edges of (unpunctuated) BOOK-rep $3_{\text {horizontal }}$ can 
also serve as antecedents for anaphora, as seen in (33)b,f; we come back below to the case of the intermediate iteration of $B O O K$-rep $3_{\text {horizontal }}$.

(33) YESTERDAY IX-1 VISIT POSS-2 APT. IX-1 SEE . POSS_DESIGN SURFACE / SURFACE DESIGN $^{27}$ FUNNY.

'Yesterday, I visited your apartment. I saw ..... looked funny.'

POSS_targets left-most $B O O K$

a. ${ }^{6.8}[\mathrm{BOOK} \text { BOOK BOOK }]_{\text {horizontal }}$ $=$ three books;.....$=$ the left-most book

b. ${ }^{6}$ BOOK-rep 3 horizontal $=$ at least three books; $\ldots . . .=$ the left-most book $(4 / 5$ judgments $)$ (or: all the books $(1 / 5$ judgment))

POSS_targets intermediate $\mathrm{BOOK}^{28}$

c. ${ }^{5.4}[\text { BOOK BOOK BOOK }]_{\text {horizontal }}$ (ratings: 6, 5, 4, 5, 7 - see Supplementary Materials for different ratings on different scenarios) $=$ three books; $\ldots . . .=$ the middle book (or possibly: all the books - see Supplementary Materials for different ratings on different scenarios)

d. ${ }^{5.2}$ BOOK-rep3 $3_{\text {horizontal }}$ (ratings: 7, 5, 3, 5, 6 - see Supplementary Materials) judgment)) $=$ at least three books; ..... = all the books $(4 / 5$ judgments) (or: a book in the middle $(1 / 5$

POSS_targets right-most $\mathrm{BOOK}$

e. ${ }^{7}[\text { BOOK BOOK BOOK }]_{\text {horizontal }}$ $=$ three books $;. . . .=$ the right-most book

f. ${ }^{6.4}$ BOOK-rep3 ${ }_{\text {horizontal }}$ $=$ at least three books; ..... = the right-most book

(ASL, 32, 0034; 5 judgments)

For reasons that we do not understand, the punctuated repetition with anaphora to the intermediate iteration is a bit degraded - something we did not find in other cases of punctuated repetitions, as in (36)c below. ${ }^{29}$ The same observation holds for the unpunctuated repetition of the sign, but as we will see below this finding turns out to be far more robust. In addition, there is an interpretive difference between the two cases: in punctuated repetitions, the possessive targeting the intermediate iteration has a singular denotation, namely the second of the three books; by contrast, the possessive targeting the second iteration of the unpunctuated repetition is preferably understood to refer to all the books.

We will argue that due to the iconic semantics of repetition-based plurals, the edges (more precisely: the vertices) of representations can introduce discourse referents because they can be inferred to have a singular denotation. The same conclusion does not hold for the second of three unpunctuated repetitions: as we will argue in Section 6.2, the intermediate iteration of an unpunctuated repetition probably has lower pictorial resolution than an edge, and cannot be taken to refer to a singular object.

This general line of analysis predicts that if we vary the shape of the unpunctuated repetition so that each of the three iterations appears at a vertex, each should be understood to denote a singular object, and should thus become a possible antecedent for a singular pronoun. This prediction is borne out, as is seen in $(34) \mathrm{c}, \mathrm{d}$, where a horizontal unpunctuated repetition is contrasted with a triangular one. In the former case, we replicate the general findings pertaining to (33)c; but in the latter case, each vertex of the triangle can be indexed by the pronoun POSS_.

${ }^{27}$ The consultant signed DESIGN SURFACE in a,b,c,d, and SURFACE DESIGN in e,f. The involuntary inversion does not affect the point under discussion here.

${ }^{28}$ When several scores were given by the consultant, averages are computed for a scenario that does not require that the books be identical, nor that all books have funny covers; see the Supplementary Materials for full data.

${ }^{29}$ An anonymous referee suggests that the phonological form of the sign might play a role: in $B O O K$ the hands move away from each other, whereas in $T R O P H Y$ they converge on a location, which may increase the identifiability of the location. We leave this issue for future research. 
(34) YESTERDAY IX-1 VISIT POSS-2 APT. IX-1 SEE TROPHY-rep-_. POSS_CARVE WORDS FUNNY.

'Yesterday, I visited your apartment. I saw several trophies, arranged in a The inscription of ...... was funny.'

POSS_targets the left-most TROPHY

a. ${ }^{6.5}$ TROPHY-rep 3 horizontal $=$ row $; \ldots . . .=$ the left-most trophy

b. ${ }^{6.5}$ TROPHY-rep $3_{\text {triangle }}$ $=$ triangle $; \ldots \ldots=$ the left-most trophy

POSS_targets the intermediate TROPHY

c. ${ }^{5.2}$ TROPHY-rep $3_{\text {horizontal }}$ $=$ row $; \ldots . .=$ all trophies $/$ the intermediate trophy

$\Rightarrow$ all the trophies are funny ( $3 / 4$ judgments) or the intermediate trophy is funny ( $4 / 4$ judgments)

d. ${ }^{6.2}$ TROPHY-rep 3 triangle $=$ triangle $; \ldots . .=$ the top trophy

$=>$ the top trophy was funny

POSS_targets the right-most TROPHY

e. ${ }^{6.5}$ TROPHY-rep3 $3_{\text {horizontal }}$ $=$ row $; \ldots . . .=$ the right-most trophy

f. ${ }^{6.5}$ TROPHY-rep 3 triangle $=$ triangle $; \ldots . .=$ the right-most trophy

(ASL, 32, 0084; 4 judgments)

As can be seen in the scores, the 'odd man out' is (34)c, where the possessive pronoun targets the middle of TROPHY-rep $3_{\text {horizontal }}$. In addition, as can be seen in the Supplementary Materials, the inferential questions gave rise to several possible answers, and in particular the possessive pronoun could be understood to refer to all trophies, or just to the middle one. By contrast, the example in (34)d was more acceptable, and the pronoun was taken to refer to the trophy at the top of the triangle.

In sum, two effects are obtained when POSS- targets the middle of an unpunctuated repetition of TROPHY arranged in a row: it becomes possible for the pronoun to denote the entire group of trophies; and the sentence becomes a bit less acceptable. There are two general directions to account for these observations.

1. The first possibility is that intermediate indexing fails to pick out a singular object, and that a rescue strategy is applied thanks to a plural reinterpretation of the pronoun. On this view, intermediate indexing is possible when TROPHY-rep3 has a triangular shape because the intermediate iteration appears at a vertex.

2. An alternative possibility is that intermediate indexing is just ambiguous, and that the ambiguity itself is to be blamed for the degraded status of the sentence. On this view, intermediate indexing fails to yield an ambiguity in the triangular case because pointing towards the top of the triangle cannot as easily be mistaken for pointing towards the entire triangle.

We find the first possibility more convincing, for two reasons (but see fn. 33 for data that might go in a different direction).

(i) First, the 'Edge Effect' arises in the paradigm in (35), with an unpunctuated (plural) repetition arranged in a row. Adding a possessive does not affect acceptability if it targets the right edge of the line, as in (35)b, but it lowers acceptability if it targets the middle of the row, as in (35)c. Importantly, in the latter case the possessive is understood to denote the middle trophy, without an ambiguity, and thus it is a bit unlikely that ambiguity per se explains the degraded character of intermediate indexing.

(35) HERE HAVE TROPHY-rep3 $3_{\text {horizontal }}$.

'Here there are several trophies arranged in a row.'

a. ${ }^{7}$ [no additional sentence]

b. ${ }^{7}$ POSS-right SHAPE STRANGE.

'The right-most trophy has a weird shape.'

$=>$ the right-most trophy has a weird shape 
c. ${ }^{5}$ POSS-middle SHAPE STRANGE.

'The intermediate trophy has a weird shape.'

$\Rightarrow>$ the intermediate trophy has a weird shape

(ASL, 33, 0592; 3 judgments)

(ii) Second, and less clearly, in simple cases of punctuated repetitions, the intermediate iteration isn't readily interpreted as referring to the entire group. Rather, a pronoun targeting the second of three punctuated copies just refers to the second of three objects, not to the entire group, as seen in (36)c. This suggests that plural reference by way of a non-plural pronoun is restricted, although one cannot exclude the possibility that plural reference is for some reason more readily available for unpunctuated repetitions than for punctuated repetitions. (On the other hand, if a plural interpretation of a non-plural pronoun is used as a rescue strategy only, we can understand why this interpretation arises simultaneously with degraded judgments in the case of unpunctuated repetitions.)

\section{HERE HAVE [TROPHY TROPHY TROPHY $]_{\text {horizontal }}$.}

a. ${ }^{7}$ [no additional sentence]

'Here there are at least three trophies [possibly in a row].'

b. ${ }^{7}$ POSS-right SHAPE STRANGE.

'Here there are at least three trophies in a row. The right-most one has a weird shape.'

$\Rightarrow$ the right-most trophy has a weird shape

c. ${ }^{7}$ POSS-middle SHAPE STRANGE.

'Here there are at least three trophies in a row. The intermediate one has a weird shape.'

$\Rightarrow>$ the intermediate trophy has a weird shape

(ASL, 33, 0596, 3 judgments)

The contrast between punctuated and unpunctuated repetitions can be further ascertained in (37), where a continuous repetition has been added to complete the paradigm; since the individual iterations are even harder to individuate in this case than in unpunctuated repetitions, we expect the judgments to be closer to those of unpunctuated than of punctuated repetitions (we do not further investigate the interaction of continuous repetitions with the Edge Effect, although we will revisit other aspects of continuous repetitions in Section 8.2). Here we asked the consultant to assess the first sentence on its own, and then to assess the entire discourse - with the goal of determining how the addition of POSS targeting the middle TROPHY influenced acceptability. The same contrasts are found as before: POSS can index the middle iteration when the repetition is punctuated; when it is unpunctuated, intermediate indexing is degraded when it targets the middle of a row but not when it targets the top of a triangle (there might be a similar effect with continuous repetitions, but these are slightly degraded to begin with, and the judgments are unstable).

Notation: 6_4.5 and other pairs of ratings are sequences of the two scores: the first (here: 6) pertains to the first sentence evaluated on its own; the second (here: 4.5$)$ pertains to the entire discourse.

(37) HERE HAVE . POSS-middle SHAPE STRANGE.

'Here there are (at least) three trophies. The intermediate one has a weird shape.'

-horizontal

a. ${ }^{7-6.7}$ TROPHY TROPHY TROPHY

b. ${ }^{7-5.3}$ TROPHY-rep

c. ${ }^{6}{ }^{4}$ TROPHY-cont

-triangular

d..$^{7} 7$ TROPHY TROPHY TROPHY

e. ${ }^{6.7-7}$ TROPHY-rep

f. 5.75 TROPHY-cont (full judgments: 5_4, 7_7, 5_4)

(ASL, 33, 0588; 3 judgments) 


\subsection{Towards an account of the Edge Effect}

We believe that the solution to this puzzle will in the end depend on a detailed understanding of the semantics of iconic representations. Two points must be understood; the first is straightforward from the perspective of an iconic semantics, the second is not.

(i) First, why can vertices introduce discourse referents? We believe that this follows from an iconic semantics (possibly along the lines of Greenberg 2013): if a picture denotes an object, modulo some constraints, parts of the picture denote parts of the object.

This makes it in principle possible to infer the existence of new discourse referents from the iconic semantics of a plural. It is worth noting that this condition would work particularly well in the case of punctuated repetitions discussed so far: BOOK BOOK BOOK as well as TROPHY TROPHY $T R O P H Y$ triggered the inference that each copy of the iterated word denoted a singular object. We initially analyzed this fact by positing that each iteration of the noun introduced a separate discourse referent, but we could have developed things in a purely iconic direction by positing that each noun was the representation of an object. At this point, the second direction is just less explicit than the first one (because it relies on ill-understood properties of iconic semantics). But we will be forced to revisit our data from this more purely iconic perspective in Section 7. As a point of comparison, suppose I ask you what you saw through a microscope, and that you draw three dots in a triangular shape $\therefore$, or in a horizontal shape .... This will provide me with some information about the arrangement of the three small things you saw. I may also ask you for details by pointing to one point or the other, and you will know which object I intended: each of the dots refers to a different object.

At this point, then, our account is based on the principle in (38).

(38) Iconic representations: downward closure

If a representation $r$ iconically represents an object $d$, and if $r$ is made of parts of $r$ that are above the

resolution of $\mathbf{r}$, then there is a part of $d$ that $r^{\prime}$ iconically represents.

The notion 'iconically represent' ought to be explicated in the end within a framework such as Greenberg 2013 , in which there is a geometric projection (which depends on the perspective adopted) between a 3D scene and a plane, one on which areas of the plane are colored differently depending on some properties of parts of the object that get projected onto them (in the case of signs, things are more complicated because the plane is replaced with a 3D space).

We immediately stated the principle with the boldfaced part ('above the resolution of $r^{\prime}$ ), pertaining to the resolution of the representation. ${ }^{30}$ To see the need for such a notion, consider the coarse-grained picture of Barack Obama in (39).

(39) Coarse-grained pictorial representation of Barack Obama's face (Jenkins and Kerr 2013)

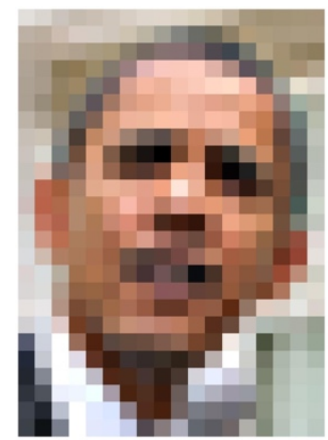

Each pixel of the central area of the picture provides information about a part of Obama's head, and one could say that each pixel $r^{\prime}$ refers to a part d' of Obama's head. But by definition subparts of a pixel $r^{\prime}$ do not reflect properties of subparts of d' because they are below the resolution of the picture. As a result, one can extrapolate what subparts of a pixel refer to, but this is not given by the mode of representation. Thus the restriction to the resolution of $\mathrm{r}$ in (38) can be explained in terms of the 'atoms'

\footnotetext{
${ }^{30}$ Work in progress by Gabe Greenberg discusses related notions.
} 
of the iconic representations, i.e. the minimal parts of the iconic representation that can be taken to have a denotation.

(ii) Our second challenge is to explain why the condition that works at the vertices of the plural representation fails for the middle of a row of unpunctuated repetitions. This is a harder puzzle to solve because it requires a deeper understanding of iconic semantics than we currently have.

One possible line of analysis is that unpunctuated repetitions offer lower resolution for the interior of objects than for their edges and vertices, presumably for reasons that have to do with properties of human perception. The image of Obama in (40) is recognizable although little information is provided about entire parts of the face; what matters is that the edges are represented, presumably because we pay greater attention to those than to non-edges. On this view, then, edges and vertices of the representation of an object are perceived with greater resolution than intermediate parts. (This line of analysis should in the end be integrated with work on object perception, for which edges are known to play a prominent role. ${ }^{31}$ )

(40) Contour Barack Obama's face (from Greenberg 2013)

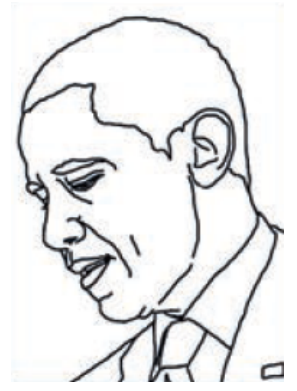

We could use this intuition to argue that in the case of TROPHY-rep $3_{\text {horizontal }}$, the first and third iterations have higher resolution than the second, somewhat as in the representation in (41)a. By contrast, in standard cases involving [TROPHY TROPHY TROPHY] $]_{\text {horizontal }}$, each iteration comes with the same resolution as the other, as in (41)b.

(41) a. Clear representation at the edges, blurred representation in the middle ${ }^{32}$

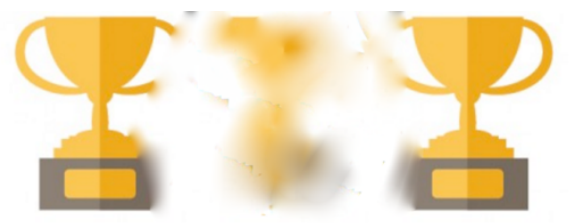

b. Clear representation throughout

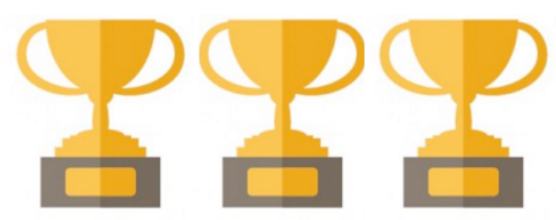

On this view, it is not clear what one points to when one points towards the middle of (41)a, or of TROPHY-rep $3_{\text {horizontal. }}$ But $w h y$ this is would need to be explained. The problem is not trivial.

We could start by postulating that the intermediate part is 'blurred' and thus can refer to any number of trophies. In effect, this would combine an iconic semantics with a vague semantics for some

${ }^{31}$ As summarized in a recent textbook on vision, "objects are defined by their edges": "we tend to ignore the variations in shading across an object. This is why a cartoon is as recognizable as a photograph. It is the edges that matter, for object perception as well as lightness perception." (Daw 2012 p. 127)

${ }^{32}$ Pictures are modified from http://www.freepik.com/free-vector/flat-trophy-collection_843005.htm\#tterm=trophy\&page=1\&position=2. 
parts of a representation. An advantage of this view is that it connects the vagueness of the quantitative threshold to the difficulty of referring by indexing the middle of the representation. But this line of analysis raises two problems.

- First, in the case of the triangular unpunctuated repetition TROPHY-rep3 $3_{\text {triangular }}$, each of the vertices could be indexed, but it didn't follow that the triangle came with a clear quantitative threshold. Thus it would seem that the denotation of the entire triangular representation is not just the sum of the denotations of the three iterations taken together. This need not be a big problem, since the characteristic of unpunctuated repetitions is that the iterations are not clearly delineated, and thus one can take the representation to also include what is between these iterations..$^{33}$

- Second, and more importantly, it is non-trivial to explain why pointing towards the intermediate iteration doesn't yield a reading on which one is referring to all the trophies that are not at the edges - something we never obtained in these examples, despite the fact that plural readings were marginally available (when we obtained a reading on which the possessive indexing the intermediate iteration referred to an object 'in the middle', it was a singular object, rather than the group of all objects not found at the edges).

We won't solve the second problem in this piece. There are at least two possible lines of analysis.

- One is that in TROPHY-rep $3_{\text {horizontal }}$, the first and the third $T R O P H Y$ refer to parts, but the intermediate iteration doesn't refer at all - although the entire unpunctuated repetition as a whole refers to a group. While in principle different parts of a representation may have different levels of resolution, as illustrated by (41)a, we would still need to explain this difference between the intermediate iteration and those that appear at edges.

- A slightly different view is that the intermediate iteration does refer (presumably to the group of objects that are not at an edge), but that pointing fails because it is not clear what is being pointed to, possibly because the intermediate iteration is blurred, and not clearly separated from its neighbors, as is illustrated in (42). On this view, then, it is not the second iteration per se that lacks a denotation, but rather the pointing sign that fails to recover that denotation.

\footnotetext{
33 Still, we cannot exclude the possibility that when each vertex is simultaneously indexed (in one and the same sentence), a precise threshold (= 'exactly three') emerges; we leave an investigation of this issue for future research. We do have one example with [TROPHY-rep 3$]_{\text {horizontal }}$ combined with three possessive pronouns, each indexing a different iteration:
}

(i) Context: In the Da Vinci Code, the main character is told by the speaker that the key to the enigma will be in a museum. Specifically:

\section{${ }^{7}$ SOMEWHERE HAVE TROPHY-rep3-horizontal. POSS-RIGHT CARVE WORDS HAVE RIGHT ANSWER. POSS-CENTER CARVE WORDS HAVE FALSE ANSWER. POSS-LEFT CARVE WORDS HAVE IRRELEVANT ANSWER.}

'Somewhere there are 3 trophies in a row. The right-most trophy's inscription has the right answer. The intermediate trophy's inscription has a false answer. The left-most trophy's inscription has an irrelevant answer.' (ASL, 32, 0144; 3 judgments)

In 2 judgments out of 3, the sentence was taken to refer to three trophies, in one judgment to at least three. In all cases, the possessive pronoun indexing the intermediate iteration was taken to refer to the trophy in the middle. In view of this particular discourse, it is unsurprising that we do not get a reading on which this pronoun refers to all trophies, as this would yield a contradiction. On the other hand, it is a bit surprising that this example is not degraded. Given its realization, we do not think that this is because the plural could be mistaken for a punctuated repetition - to Lamberton, it is clearly unpunctuated. 
(42) Successful pointing (on the left) and unsuccesful pointing (in the middle)

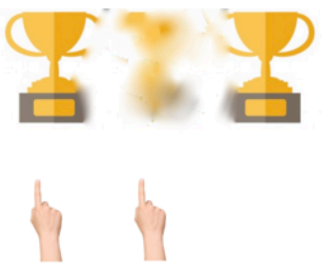

It will take a more articulated iconic semantics to solve this problem. But we will see in Section 10 that similar generalizations could in principle be tested with some iconic gestures in spoken language.

\subsection{Other iconically inferred discourse referents}

It is worth noting that at least one other case of iconically inferred discourse referents has been discussed in the literature. In ASL (and LSF), plural loci are usually realized as (semi-)circular areas. These can be embedded within each other, and when a small plural locus $a$ denoting a group s(a) appears within a large locus $a b$ denoting a group $\mathrm{s}(\mathrm{ab})$, a 'complement set' locus $b$ suddenly comes into existence, and denotes $s(a b)-s(a)$.

The argument was developed by studying examples such as (43), where the large locus $a b$ denotes the set of all students, while a sub-locus $(=a)$ denotes the set of students that came (Schlenker et al. 2013). The point is that a complement locus $(=b)$ thereby becomes available, denoting the set of students who didn't come, as illustrated in (44).

(43) POSS-1 STUDENT IX-arc-ab MOST IX-arc-a a-COME CLASS.

'Most of my students came to class.'

a. ${ }^{7}$ IX-arc-b b-STAY HOME.

'They stayed home.'

b. ${ }^{7}$ IX-arc-a a-ASK-1 GOOD QUESTION.

'They asked me good questions.'

c. ${ }^{7}$ IX-arc-ab SERIOUS CLASS.

'They are a serious class.'

(ASL, 8, 196; Schlenker et al. 2013)

(44)

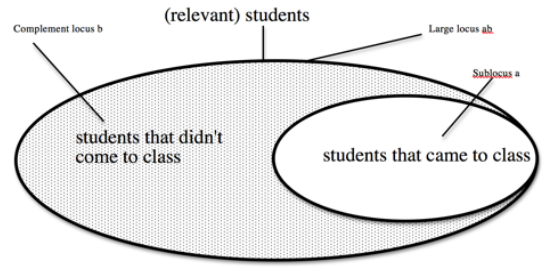

The readings obtained with (43)b,c could be replicated with standard anaphora in English, as shown by (45)b,c. But the reading obtained in (43)a is more surprising, since it cannot be obtained by standard anaphoric mechanisms in English, as shown in (45)a. ${ }^{34}$ Furthermore, the English data can be replicated in ASL when a single default is used, as illustrated in (46), which is deviant.

${ }^{34}$ This reading can be obtained with the expression the others, which is not a pronoun, unlike the expressions in (43) and (45). A plausible analysis of the others is that it has an anaphoric index, so that the others $s_{i}$ is interpreted as the (salient) group different from $i$. In (i), $i$ can be taken to refer to the set of students that came to class, just as in (45)b.

(i) Most students came to class. The others $\mathrm{s}_{\mathrm{i}}$ stayed home instead. 
(45) a. \#Most students came to class. They stayed home instead.

b. Most students came to class, and they asked good questions.

c. Most students came to class. They are a serious group.

(46) ${ }^{2.8}$ POSS-1 STUDENT MOST a-CAME CLASS. IX-arc-a a-STAY HOME.

Intended: 'Few/Most of my students came to class. They [the students that didn't come] stayed home.'

(ASL, 8, 200; Schlenker et al. 2013)

Nouwen 2003 argues that the unavailability of (45)a shows that although the first sentence makes available a discourse referent denoting the students that came to class, and possibly also one that refers to the entire group of students, no discourse referent is made available to refer to the students that did not come to class. Schlenker et al. 2013 extend Nouwen's observation to ASL to account for (46), but this makes it all the more surprising that (43)a is acceptable.

The proposal in Schlenker et al. 2013 is that this reading is made available by a mechanism of iconically inferred discourse referents. The main assumptions are that (i) the set of loci is closed with respect to relative complementation: if $a$ is a sublocus of $b$, then $(b-a)$ is a locus as well; and (ii) assignment functions are constrained to respect inclusion and relative complementation: if $a$ is a sublocus of $b$, the denotation of $a$ is a subpart of the denotation of $b$, and $(b-a)$ denotes the expected complement set. Both principles applied to the situation depicted in (44) guarantee that the complement locus $b$ turns out to denote the students that didn't come to class.

While this is a more abstract (diagrammatic) sort of iconicity than the case of unpunctuated repetitions, some key principles apply in both domains. First, if a complex iconic representation $r$ denotes a group g, a subpart $r^{\prime}$ of $r$ may denote a subpart $g^{\prime}$ of $g$. Second, the choice of subparts that are thus endowed with reference is constrained: once a locus and a sublocus are established, the only third discourse referent that becomes available is the complement set locus; similarly, we saw above that edges of an unpunctuated plural are endowed with singular reference more easily than other parts. Third, the interpretation 'preserves' some geometric properties holding between an iconic representation and its parts: if $r^{\prime}$ is a sublocus of $r^{\prime}$, the denotation of $r^{\prime}$ should be a subgroup of the denotation of $r$; if $r^{\prime}$ is at an edge of an unpunctuated repetition $r$, the denotation of $r^{\prime}$ should be at an edge of the denotation of $r$. A comparison between these two instances of iconicity with inferred discourse referents is sketched in (47).

a. Structural iconicity in Schlenker et al. 2013: subset and relative complementation relations among semi-circular loci are preserved by assignment functions (written as $s$ ).

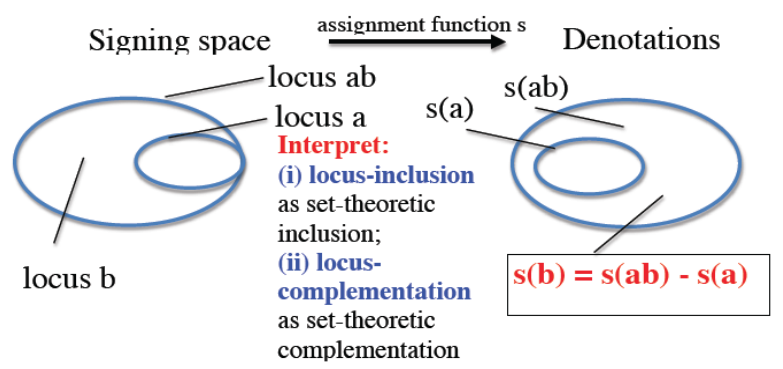

b. Iconicity of nominal plurals as unpunctuated repetitions of a sign: here the 3 iterations $a b c$ of the sign denote a group (containing more than 3 objects), but the horizontal repetition of the sign indicates that the denoted group is horizontal, and the edges of the sign denote the edges of the group (here the interpretation function is written as $I$ ).

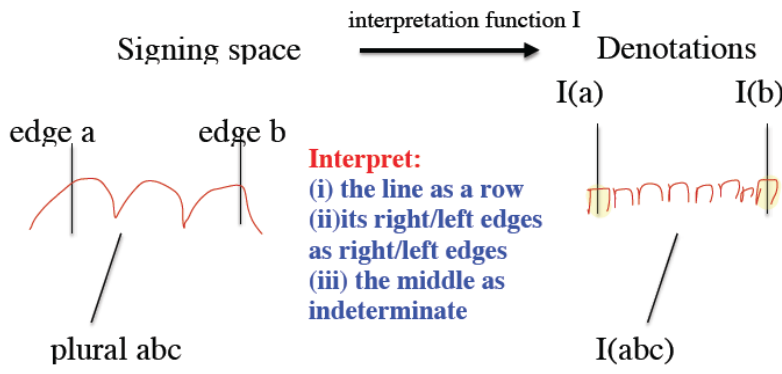

\subsection{Neutral vs. iconic forms}

It is an open question whether punctuated or unpunctuated repetitions can be interpreted non-iconically, i.e. without providing any information about the arrangement of the denoted entities. If such neutral interpretations are available, they could have two sources: it could be that a default arrangement of the repeated signs is interpreted as being intrinsically non-iconic, as plurals are in English; or it could be 
that some iconic interpretations are so vague as to provide little positional information. ${ }^{35}$ In the second but not in the first case, one would expect some vague iconic information to be provided by the repetition.

Be that as it may, it is worth observing that the Edge Effect discussed in the previous sections leads us to expect that a 'neutral' form should be unacceptable when combined with anaphora to an edge, or that it should be reinterpreted as iconic. ${ }^{36}$ The reason is that, for us, it is because of their iconic semantics that edges can come to create singular discourse referents.

The paradigm in (48) tests this correlation, but the results are not conclusive. The punctuated and unpunctuated repetitions of SENTENCE appear in a row. The first sentence evaluated on its own only provides weak iconic information. Adding the second clause with a right-edge possessive pronoun strengthens the iconic inference in (48)a and (48)c, but the effects are not entirely clear (see the Supplementary Materials for details). ${ }^{37}$

(48) YESTERDAY YOU WRITE MEANING VAGUE.

'Yesterday you wrote sentences. The meaning of the right-most sentence/of all the sentences was vague.'

a. ${ }^{6.7} \ldots=[\text { SENTENCE SENTENCE SENTENCE }]_{\text {horizontal }} \quad \ldots=$ POSS_right

$2 / 3$ judgments: the sententences are more likely to be in a row when the 2nd clause is added

b. ${ }^{7}-=[\text { SENTENCE SENTENCE SENTENCE }]_{\text {horizontal }} \quad \ldots=$ POSS_arc

$0 / 3$ judgment: the sentences are more likely to be in a row when the 2nd clause is added c. ${ }^{6.3}$ __ $=$ SENTENCE-rep3 3 horizontal $\quad \ldots=$ POSS-right

$3 / 3$ judgments: the sentences are more likely to be in a row when the 2 nd clause is added

d. ${ }^{7} \ldots=$ SENTENCE-rep 3 horizontal $\quad \ldots=$ POSS-arc

$1 / 3$ judgment: the sentences are more likely to be in a row when the 2 nd clause is added (ASL, 34, 2256; 3 judgments)

\section{Punctuated repetitions revisited}

We now return to punctuated repetitions, and explain why it would be incorrect to treat them as conjoined indefinites. We argue that it is because of their iconic semantics that punctuated repetitions introduce different discourse referents, and not because they are made of separate words.

\subsection{Punctuated repetitions with numerals}

The heart of the matter is that if we treat a punctuated repetition such as [TROPHY TROPHY TROPHY] as three conjoined indefinites, this expression should not co-occur with any numeral but 3 . In fact, if we liken this expression to a trophy and a (second) trophy and a (third) trophy, we wouldn't expect any numeral to co-occur with it. But in our data such a co-occurrence is deemed rather acceptable, be it with the numerals 3,4 or 10 . This is most clearly seen in the paradigm in (49), where the boldfaced expressions in (49)f, h display precisely the co-occurrence we don't expect. Importantly, the iterations were all signed as an arc on the horizontal plane. When the repetition was unpunctuated, this could also

\footnotetext{
${ }^{35}$ It is worth noting that sign modulations and facial expressions might conceivably be used to indicate that an iconic representation is imprecise. The preliminary data we collected are not conclusive yet (ASL 34, 2264).

${ }^{36}$ Thanks to Masha Esipova for urging that we revisit this point.

${ }^{37}$ Two remarks should be added.

1. In a paradigm (ASL, 34, 2258) in which the iterations of SENTENCE are arranged vertically rather than horizontally, a vertical plural possessive pronoun suffices to trigger the iconic inference that the sentences are arranged vertically (as in a list or essay); the same effect is obtained if a singular possessive pronoun targets the top of the list. When there is no possessive pronoun, or a horizontal plural possessive pronoun, iconic information is weak, indicating only that the sentences are probably not in a row.

2. Iteration of SENTENCE within a single location is possible. For Lamberton, the repetition tends to yield different meanings depending on how it is realized. If the repetitions are realized without interval (hence like unpunctuated repetitions), he obtains a meaning akin to 'many sentences'. With a significant interval between the iterations (which might make them punctuated), the meaning still involves many sentences, but more time is involved in the relevant action. We leave this question for future research, but note that a comparison with repetition-based pluractionals (as studied in particular by Kuhn 2015 and Kuhn and Aristodemo 2017) would be particularly interesting.
} 
be taken to be a horizontal triangle since only three points were displayed (with the tip of the triangle towards the addressee). The use of a punctuated repetition provides iconic information to the effect that the trophies are 'spread out', whereas the unpunctuated repetition indicated that they were 'all around' (sometimes arrangement as an arc was mentioned, as seen in the Supplementary Materials).

(49) Unmarked form of TROPHY

MUSEUM HAVE

a. ${ }^{7}$ TROPHY-rep3 $3_{\text {horizontal_arc. }}$

'The museum has trophies (all around).'

b. ${ }^{7}$ [TROPHY TROPHY TROPHY $]_{\text {horizontal__arc }}$.

'The museum has 3 trophies (spread out).'

c. ${ }^{5.7} 3$ TROPHY-rep4 4 horizontal_arc.

'The museum has 3 trophies (all around).'

d. ${ }^{7} 3$ [TROPHY TROPHY TROPHY] $]_{\text {horizontal_arc. }}$

'The museum has 3 trophies (spread out).'

e. ${ }^{6.7} 4$ TROPHY-rep4 4 horizontal_arc.

'The museum has 4 trophies (all around).'

f. ${ }^{7} 4$ [TROPHY TROPHY TROPHY $]_{\text {horizontal_arc }}$.

'The museum has 4 trophies (spread out).'

g. ${ }^{7} 10$ TROPHY-rep4 $4_{\text {horizontal_arc. }}$

'The museum has 10 trophies (all around).'

h. ${ }^{7} 10$ [TROPHY TROPHY TROPHY] $]_{\text {horizontal_arc. }}$

'The museum has 10 trophies (spread out).'

(ASL 34, 2216; 3 judgments)

The same point is made by the paradigm in (50), which primarily differs from (49) in that a nominal form of TROPHY is used, involving one repetition in the singular. As was mentioned earlier, the distinction between nominal and unmarked forms seems to be neutralized in the (unpunctuated) plural, which normally uses the unmarked form - with the result that the attempt to create an unpunctuated repetition of the nominal form leads to a slight deviance, as in (50)a,c,e,g. What matters for our purposes is that the punctuated repetitions are acceptable with numerals, in particular 4 and 10 in the boldfaced examples in (50)f,h. Here too, iconic information is provided to the effect that the trophies are spread out or all around (the consultant sometimes mentioned more precise iconic information involving arc-shaped arrangements; see the Supplementary Materials).

(50) Nominal form of TROPHY $Y^{38}$

MUSEUM HAVE

a. ${ }^{6}$ TROPHYn-rep 3 horizontal_arc.

'The museum has trophies all around.'

b. ${ }^{7}$ [TROPHYn TROPHYn TROPHYn $]_{\text {horizontal_arc }}$.

'The museum has 3 trophies spread out.'

c. ${ }^{5.3} 3$ TROPHYn-rep3 ${ }_{\text {horizontal_arc. }}$.

'The museum has 3 trophies all around.'

d. ${ }^{7} 3$ [TROPHYn TROPHYn TROPHYn $]_{\text {horizontal_arc }}$.

'The museum has 3 trophies spread out.'

e. ${ }^{6} 4$ TROPHYn-rep 3 horizontal_arc.

'The museum has 4 trophies all around.'

f. ${ }^{7} 4$ [TROPHYn TROPHYn TROPHYn] $]_{\text {horizontal_arc. }}$

'The museum has 4 trophies spread out.'

g. ${ }^{5.7} 10$ TROPHYn-rep3 $3_{\text {horizontal_arc. }}$

'The museum has 10 trophies all around.'

h. ${ }^{7} 10$ [TROPHYn TROPHYn TROPHYn] $]_{\text {horizontal_arc. }}$

'The museum has 10 trophies spread out.'

(ASL, 34, 2218; 3 judgments)

\footnotetext{
${ }^{38}$ In 1/3 judgment, the consultant mentioned an additional possible reading for (50)c,e,f,h, involving 3, 4 or 10 groups of trophies. See the Supplementary Materials for details (judgments dated [JL 16.12.05]).
} 


\subsection{Revised analysis}

We conclude that punctuated repetitions should not be analyzed as conjoined indefinites. Upon reflection, we believe that the data in (49) and (50) point towards a more parsimonious theory: punctuated and unpunctuated repetitions have the same logical semantics, and the differences between them follow from their iconic semantics. This alternative theoretical direction is sketched in (51) ${ }^{39}$

(51) a. Punctuated and unpunctuated repetitions have the same logical semantics but different iconic semantics. (Either because of the logical or because the iconic semantics, each comes with an existential condition to the effect that there is a plurality of objects satisfying a certain condition, which may be precise or vague.) b. Unpunctuated repetitions

(i) Because part of an unpunctuated repetition has low resolution (it is not the case that each iteration necessarily denotes one object), conditions on the number of denoted objects are not precise.

(ii) Numerals can be added to specify the number of denoted objects.

(iii) Because of their iconic semantics, vertices can introduce (iconically inferred) discourse referents.

(iv) Because the iconic representation has lower resolution outside of vertices, no singular discourse referents are introduced by non-vertices.

\section{c. Punctuated repetitions}

(i) Because each iteration has high resolution, each is preferably interpreted as denoting one object.

(ii) Numerals can override this preference, in which case the interpretation specifies that the denoted objects are clearly separable: the punctuated nature of the repetition is taken to represent a salient aspect of the denoted situation.

(iii) When the interpretation makes it possible to assign one singular object to each iteration, all iterations (not just vertices) introduce (iconically inferred) discourse referents.

On this unified view, the semantics of both constructions can be given as in (52), and it is left to the iconic semantics of each construction to specify precise or vague conditions on the number of objects denoted, as well as the singular discourse referents that can be inferred from the iconic semantics of the construction. (On a technical level, it is worth noting that $B O O K$-iter $_{X} P$ evaluated under an assignment s causes $P$ to be evaluated under a modified assignment that assigns a new value to the plural variable $X$ [as is standard], but also to singular discourse referents $x_{1} \ldots x_{n}$ whose denotations can be inferred on iconic grounds. Note that we will further refine this analysis in Section 9.)

(52) Semantics of punctuated and unpunctuated repetitions (to be revised)

For $B O O K-$ iter $_{X}=B O O K-r e p_{X}$ or $[B O O K B O O K B O O K]_{X}$, if $\mathrm{c}$ is a context, $\mathrm{s}$ an assignment function, and if $P$ a propositional expression, [[BOOK-iter $\mathrm{P}]]^{\mathrm{c}, \mathrm{s}}=$ true

iff for some plural object $\mathrm{d}, *\left[\mathrm{bbok}^{\mathrm{c}, s[\mathrm{X} \rightarrow \mathrm{d}]}(\mathrm{d})=\right.$ true and $\boldsymbol{B O O K}$-iter ${ }_{X}$ iconically represents $\mathrm{d}$ given $\mathrm{c}$

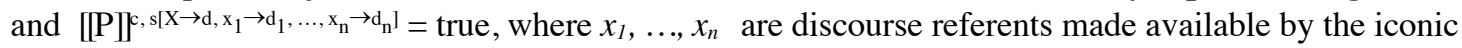
semantics of BOOK-iter $x$, and $x_{1}, \ldots, x_{n}$ respectively denote $\mathrm{d}_{1}, \ldots, \mathrm{d}_{\mathrm{n}}$ (which are parts of $\mathrm{d}$ ),

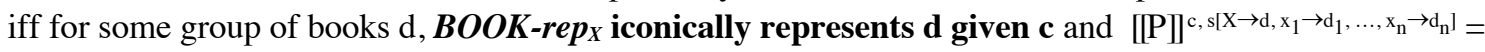
true.

On this analysis, we expect the threshold introduced by repetitions to be at least the number of iterations that can be distinguished from each other. While we haven't specifically tested this hypothesis, the thresholds we found above are generally consistent with this prediction.

As things stand, an obvious weakness of this analysis is that much work is done by the iconic component, whose detailed properties are not well understood yet. This seems to us to be inevitable in view of the iconic nature of the construction. Still, some predictions are worth discussing.

\footnotetext{
${ }^{39}$ Throughout, we use the term 'high resolution' rather than 'salience', which might be more common in a linguistic context. This is because we take a part of an iconic representation to have high resolution if small parts of it have a denotation (this is not the case in the pixelized picture of Obama in (39), as subparts of the pixelized squares do not have an independent iconic denotation). By contrast, salience is a property of denotations rather than of representations. High resolution may but need not make the denotations of the parts salient. Thus we expect a punctuated repetition with numerous iterations $($ say, 5$)$ to have high resolution without necessarily making salient each of the denotations.
} 
- First, could the Edge Effect obliterate the semantic distinction between punctuated and unpunctuated repetitions in case each iteration is at a vertex ( $^{40}$ We saw in (34)b,d,f that a triangular unpunctuated repetition makes three singular discourse referents available, which is precisely what we expect for a punctuated repetition with three iterations. But even under these conditions, there should remain differences: for an unpunctuated repetition, what is found between the vertices should be iconically vague, whereas it shouldn't be for a punctuated repetition. We obtained such a contrast in our initial paradigm involving trophies in (28): (28)b involved a triangular-shaped punctuated repetition, (28)f a triangular-shaped unpunctuated repetition, and unlike the former the latter came with a vague quantitative threshold condition. But the comparison is not quite minimal, because (28)f involved 3 vertices but at least 4 iterations, whereas (28)b included exactly 3 iterations. By contrast, (28)d involves 3 unpunctuated, triangular-shaped repetitions. We expect it to come with less precise threshold conditions than (28)b. While the raw data in the Supplementary Materials might be indicative of some vagueness for (28)d, it seems to pertain to shape rather than to quantity. In any event, our data are not sufficiently fine-grained to fully test this prediction, which should be investigated in future research.

- Second, could the addition of a numeral to a punctuated repetition make it more similar to an unpunctuated repetition? We stated in (51)c(ii) that numerals can override the preference for having each punctuated iteration denote a separate object. But if this is so, we might expect that an edge effect might emerge even in punctuated repetitions. Our data on this matter are equivocal. A paradigm (ASL 32, 0156) involving the expression 7 [TROPHY TROPHY TROPHY] $]_{\text {horizontal }}$ detected an acceptability distinction between indexing the middle iteration (degraded) and indexing the entire group by way of a plural (arc) pronoun (acceptable). A more systematic paradigm (ASL, 34, 2236) with the expression 9 [TROPHY TROPHY TROPHY] $]_{\text {horizontal }}$ and a comparison between indexing the middle vs. the edges failed to detect a contrast (all were acceptable). We leave this question for future research.

\section{Iconic Mass Terms in ASL}

\subsection{Variation in iconic potential}

As we saw in connection in (8)c and (8)e, some mass terms can be repeated in signing space, with different readings depending on whether the repetition is continuous or not: a mass reading (with iconic conditions) is obtained with a continuous repetition; with a discontinuous repetition, a plural reading is obtained (also with iconic conditions). This conclusion was confirmed by the possible co-occurrence of a discontinuous repetition with the determiner $A-F E W$ in (9)c.

Still, it is important to note that not all mass terms are equally suited to iconic repetition. Differences across lexical items might be due to a combination of phonological and semantic factors, a topic we leave for future research. An example of a contrast between an iconic-friendly and an iconicunfriendly mass term is given in (53): $S A L T$ is iconic-friendly (as shown by the high ratings in (53)c,e), while FLOUR isn't (as shown by the low ratings in $(53) \mathrm{d}, \mathrm{f})$. No obvious semantic difference comes to mind, but it might be that the realization of FLOUR (involving an $F$ circling on the non-dominant hand) makes continuous repetition difficult to realize.

Notation: TWO-WINGS_^introduces two wings on a horizontal plane, with their tip in front of the signer; this is represented as $\wedge$ (but the reader should keep in mind that the triangular shape is signed horizontally, not vertically). SALT_/ and FLOUR_/ are continuous iterations of SALT and FLOUR respectively in the area corresponding to the left wing (from the signer's perspective), while $S A L T \_\wedge$ and $F L O U R \_\wedge$ represent iterations throughout the two wings.

\section{(53) SCIENCE LAB TWO-WINGS_^ YESTERDAY BRIEF LEAK. NOW FINISH REPAIR. BUT NOW}

STILL HAVE

'The science lab with two wings yesterday had a brief leak that is now repaired. But now there still is a. ${ }^{7}$ SALT.

salt.'

${ }^{40}$ Thanks to Malte Zimmermann for raising this question. 


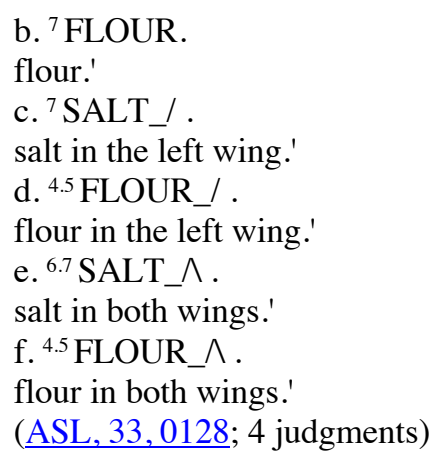

Still, there are other nouns whose form would be compatible with a continuous repetition, but which do not easily lend themselves to it. Thus the trembled $O$ used for OXYGEN seems to differ minimally from the trembled $T$ that realizes TOILET: the latter lends itself to an iconic repetition, the former doesn't. Given out current data, it would seem that, besides SALT, SMOKE (which appears in a nominal form in (54)) lends itself to rich iconic modulations, as do PEE and to some extent $A L C O H O L$, while FLOUR, OXYGEN and HYDROGEN don't. The source of these contrasts should be investigated in future research. Relevant contrasts are found in the examples in (54)-(56), which follow the same notational conventions as (53). In each case, the c. and e. examples involve a mass term which is acceptable with iconic repetition, while the $\mathrm{d}$. and f. examples involve a contrasting mass term which is degraded with iconic repetition (the $\mathrm{a}$. and b. examples are baselines without repetition).

(54) SCIENCE LAB TWO-WINGS_^Y YESTERDAY BRIEF LEAK. NOW FINISH REPAIR. BUT NOW STILL HAVE

'The science lab with two wings yetserday had a brief leak that is now repaired. But now there still is a. ${ }^{7}$ SMOKE.

smoke.'

b. ${ }^{7} \mathrm{OXYGEN.}$

oxygen.'

c. ${ }^{7}$ SMOKE_/.

smoke in the left wing.'

d. ${ }^{4.3}$ OXYGEN_/ .

oxygen in the left wing.'

e. ${ }^{7}$ SMOKE_^.

smoke in both wings.'

f. ${ }^{4.7}$ OXYGEN_ $\wedge$.

oxygen in both wings.'

(ASL, 33, 0112; 3 judgments)

(55) SCIENCE LAB TWO-WINGS_^ YESTERDAY BRIEF LEAK. NOW FINISH REPAIR. BUT NOW STILL HAVE.

'The science lab with two wings yesterday had a brief leak that is now repaired. But now there still is a. ${ }^{7}$ PEE.

pee.'

b. ${ }^{7}$ ALCOHOL.

alcohol.'

c. ${ }^{6.7}$ PEE $/$.

pee in the left wing.'

d. ${ }^{5.3}$ ALCOHOL_/.

alcohol in the left wing.'

e. ${ }^{6.3}$ PEE $\_$.

pee in both wings.'

f. ${ }^{5.3}$ ALCOHOL $\_$.

alcohol in both wings.'

(ASL, 3, 0118; 3 judgments)

(56) SCIENCE LAB TWO-WINGS_^ YESTERDAY BRIEF LEAK PEE (a, c, e ) / LIQUID HYDROGEN $(b, d, f)$. NOW FINISH REPAIR. BUT NOW STILL HAVE 
'The science lab with two wings yesterday had a brief leak of pee/liquid hydrogen that is now repaired. But now there still is

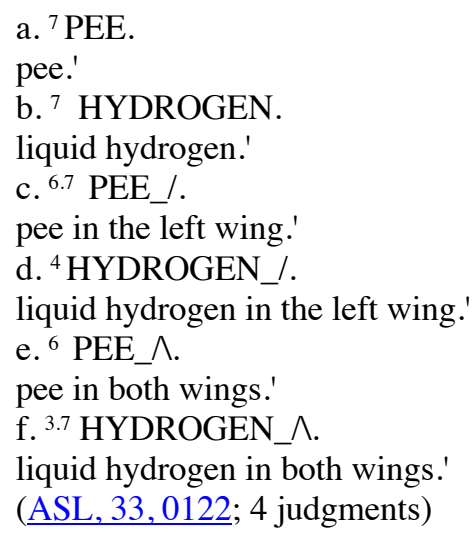

\subsection{Types of repetitions: continuous, unpunctuated, punctuated}

\subsubsection{Mass terms}

As noted above, mass terms that lend themselves to iconic modulations can be repeated continuously. This triggers the inference that the relevant substance covers an entire area without discontinuity, as can be seen in (57)c. But in addition, iconic uses can give rise to punctuated and unpunctuated repetitions, in which case one obtains readings that involve clusters: disparate and clearly distinguishable clusters in the case of punctuated repetitions, and a group of them in the case of unpunctuated repetitions, as can be seen in (57)a,b.

(57) HERE HAVE

'Here there

a. ${ }^{6.7}[\text { PEE PEE PEE }]_{\text {horizontal }}$.

are three (?) areas of pee.'

$=>$ there are (at least three?) separate areas of pee

b. ${ }^{7}$ PEE-rep horizontal.

are areas of pee.'

$\Rightarrow>$ there are (at least three?) areas of pee, possibly closer to each other than in a.

c. ${ }^{7}$ PEE-cont horizontal. $^{\text {. }}$

is a large area of pee.'

$\Rightarrow>$ there is pee in a large area

(ASL, $\underline{33,0526}$; 3 judgments)

\subsubsection{Comparison with plurals}

While our earlier discussion of count terms focused on punctuated and unpunctuated repetitions, continuous repetition is sometimes possible as well. Like unpunctuated repetition, it does not provide clear quantitative thresholds. But whereas unpunctuated repetition suggests that the denoted objects are not all clustered together, continuous repetition does not trigger this inference, as shown in (58)b,c. The comparison with (57)b,c is particularly minimal, since (as mentioned) the manual signs for PEE and TOILET are identical: for our consultant, the two words are distinguished by their non-manual components, in particular the labialization.

\section{(58) HERE HAVE}

'Here there

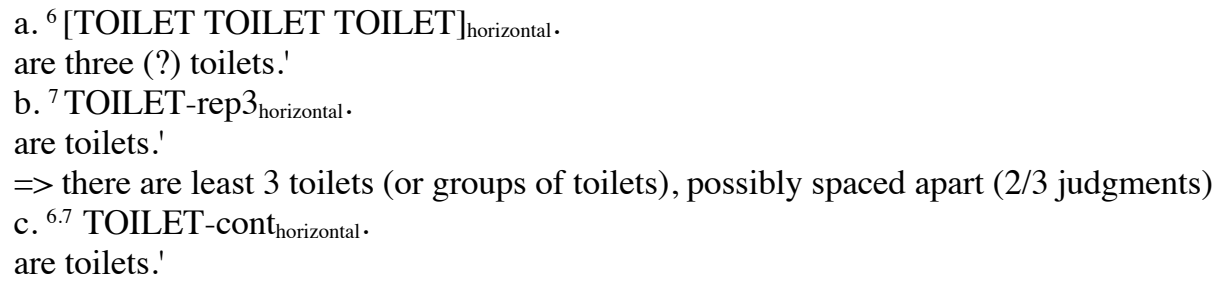


$=>$ there are at least 3-4 toilets arranged in a row (3/3 judgments)

(ASL 33, 0522a,b,c; 3 judgments)

\subsection{Dependent mass terms?}

Interestingly, mass terms give rise to a construction reminiscent of the dependent-like plurals discussed in Section 4.3. In (59)c, BLOOD-rep circular $_{\text {is }}$ iterated (which we write as -rep rather than -cont because the articulation of the sign does not clearly allow for continuous repetition) within the same area established by PEE-cont $t_{\text {circular. }}$. It is worth noting that this example involves the quantifier MOST, which does not allow for cumulative readings with plurals. Still, the reading obtained is not that each part of urine has a circular area of blood in it. Rather, the urine arranged in a circular area has blood in it arranged in the same shape.

(59) SCIENCE LAB YESTERDAY BRIEF LEAK PEE . TODAY IX-1 LOOK-GROUND MOST

a. ${ }^{7}$ PEE HAVE BLOOD.

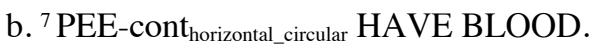

c. ${ }^{6.5}$ PEE-cont horizontal_circular HAVE BLOOD-rep $_{\text {horizontal_circular. }}$.

'The science lab yesterday had a brief leak of pee. Today I looked, most of the pee had blood in it.'

$\Rightarrow$ blood is all over the floor / throughout the puddle of urine

(ASL, 33, 0188; 2 judgments)

Importantly, the repetition-based construction with dependent-like mass terms provides iconic information about the way in which the two substances are intertwined. This is made particularly clear by the paradigm in (60): depending on where $B L O O D$-rep is signed, we obtain an inference that the blood is found throughout the urine, or towards the left, the right, the center, or both the left and the right (some examples appear to be a bit degraded because the area defined by iconic BLOOD did not cover most of the area defined by iconic PEE, contrary to what is asserted).

(60) SCIENCE LAB YESTERDAY BRIEF LEAK PEE . TODAY IX-1 LOOK-GROUND MOST

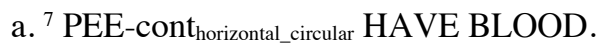

b. ${ }^{6.5}$ PEE-cont horizontal_circular HAVE BLOOD-rep $_{\text {horizontal_circular }}$.

$=>$ blood is all over the floor / throughout the puddle of urine

c. ${ }^{6}$ PEE-cont horizontal_circular HAVE BLOOD-rep $_{\text {horizontal_circular-left_only. }}$.

$=>$ blood is found in the left-hand part of the puddle of urine

d. ${ }^{6}$ PEE-cont horizontal_circular HAVE BLOOD-rep $_{\text {horizontal_circular-right_only. }}$.

$=>$ blood is found in the right-hand part of the puddle of urine

e. ${ }^{6.5}$ PEE-cont horizontal_circular HAVE BLOOD-rep $_{\text {horizontal_circular-center_only. }}$.

$\Rightarrow$ blood is found in the central part of the puddle of urine

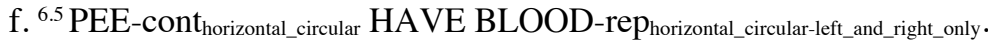

$=>$ blood is found separately in the left-hand part and right-hand parts of the puddle of urine

'The science lab yesterday had a brief leak of pee. Today I looked, most of the pee had blood in it [in specified areas as explained above for $\mathrm{b}$ through $\mathrm{f}] . '$

(ASL, 33,0196; 2 judgments)

Three conclusions can be drawn.

(i) The dependent-plural construction has a counterpart with mass terms, including in an environment (under MOST) where it is unlikely that a cumulative reading is responsible for the observed truth conditions.

(ii) In the paradigms in (59) and (60), there is a clear iconic contribution of the relative arrangement of the two mass terms. This need not be a problem for our proposal if the iconic analysis it relies

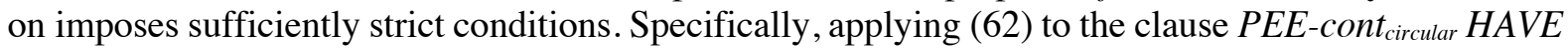
$B L O O D$-rep (or a more complicated one with MOST), we will obtain the truth conditions in (61)b, where we have disregarded the issue of 'new' discourse referents created by the iconic semantics, and assumed a Logical Form as in (61)a'.

(61) a. PEE-cont horizontal_circular HAVE BLOOD-rep $_{\text {area }}$.

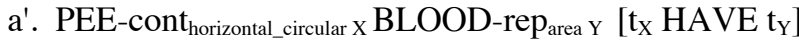

b. $\left[\left[\left(a^{\prime}\right)\right]^{c, s}=\right.$ true 


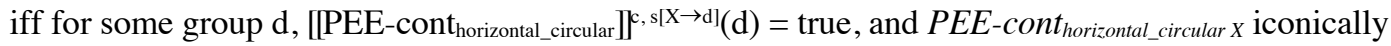
represents d given $\mathrm{c}$, and [[BLOOD-reparea $\left.\mathrm{Y}\left[\mathrm{t}_{\mathrm{X}} \mathrm{HAVE} \mathrm{t}_{\mathrm{Y}}\right]\right]^{\mathrm{c}, \mathrm{s}[\mathrm{X} \rightarrow \mathrm{d}]}=$ true,

iff for some group $\mathrm{d}$, for some group d', [[PEE-cont horizontal_circular $]^{\mathrm{c}, \mathrm{s}[\mathrm{X} \rightarrow \mathrm{d}]}(\mathrm{d})=$ true, and PEE-cont horizontal_circular ${ }_{X}$ iconically represents d given c, [[BLOOD-rep $\left.\left.{ }_{\text {area }}\right]\right]^{\mathrm{c}, \mathrm{s}\left[\mathrm{X} \rightarrow \mathrm{d}, \mathrm{Y} \rightarrow \mathrm{d}^{\prime}\right]}(\mathrm{d})=$ true, and $B L O O D$-rep area $Y$ iconically represents d' given $\mathrm{c}$, and $\left[\left[\mathrm{t}_{\mathrm{X}} \mathrm{HAVE} \mathrm{t}_{\mathrm{Y}}\right]\right]^{\mathrm{c}, \mathrm{s}\left[\mathrm{X} \rightarrow \mathrm{d}, \mathrm{Y} \rightarrow \mathrm{d}^{\prime}\right]}=$ true,

iff for some group $\mathrm{d}$, for some group $\mathrm{d}$, $\mathrm{d}$ is urine and $P E E$-cont horizontal_circular $X_{X}$ iconically represents $\mathrm{d}$ given $\mathrm{c}, \mathrm{d}^{\prime}$ is blood and BLOOD-rep area $Y_{Y}$ iconically represents $\mathrm{d}^{\prime}$ given $\mathrm{c}$, and $\mathrm{d}$ has $\mathrm{d}$ '.

Now crucially, we want the two conditions PEE-conthorizontal_ircular $X$ iconically represents $d$ given $c$ and $B L O O D$-rep area Y iconically represents $d^{\prime}$ given $c$ to jointly entail that $\mathrm{d}$ and $\mathrm{d}^{\prime}$ are intertwined in the same way as the expressions that denote them. This result should be derived by a more precise iconic semantics than we are able to develop in this piece.

Finally, one would expect that the same kind of iconic conditions could be found relating to plurals rather than mass terms - something that could be explored in future research.

\section{Towards a unified account of punctuated, unpunctuated and continuous repetitions}

Having analyzed the main properties of punctuated, unpunctuated and continuous repetitions in their interaction with iconicity, we explore prospects for a unified analysis.

\subsection{A first attempt}

In (52), we proposed a semantics for unpunctuated and punctuated repetitions. We could seek to extend it to mass terms by building on the observation that plurals and mass terms have very much the same semantics, with the difference that plurals quantify over groups that have atoms whereas mass terms do not have this constraint (for a more sophisticated discussion, see for instance Nouwen 2015, Nicolas 2016 and especially Chierchia 2010). We thus propose the partly unified account sketched in (62), where we take unpluralized count terms to be true of atoms.

(62) Semantics of punctuated, unpunctuated and continuous repetitions (initial attempt)

For $N$-iter ${ }_{X}=N$-rep $p_{X}$ or $[N N N]_{X}$ or $N$-cont , $_{X}$, if $P$ is a propositional expression,

$\left[\mathrm{N} \text {-iter } \mathrm{X}_{\mathrm{X}} \mathrm{P}\right]^{\mathrm{c}, \mathrm{s}}=$ true iff for some group $\mathrm{d}$,

(i) if $N$ is count, $*\left[[N]^{c, s[X \rightarrow d]}(d)=\right.$ true; and if $N$ is mass, $[[N]]^{c, s[X \rightarrow d]}(d)=$ true, and

(ii) $\mathbf{N}$-iter iconically represents $\mathrm{d}$ given $\mathrm{c}$, and

(iii) $\left[[\mathrm{P}]^{\mathrm{c}, \mathrm{s}\left[\mathrm{X} \rightarrow \mathrm{d}, \mathrm{x}_{1} \rightarrow \mathrm{d}_{1}, \ldots, \mathrm{x}_{\mathrm{n}} \rightarrow \mathrm{d}_{\mathrm{n}}\right]}=\right.$ true, where $x_{1}, \ldots, x_{n}$ are discourse referents made available by the iconic

semantics of $N$-iter, and $x_{1}, \ldots, x_{n}$ respectively denote $\mathrm{d}_{1}, \ldots, \mathrm{d}_{\mathrm{n}}$ (which are parts of $\mathrm{d}$ ).

In all cases, then, the relation 'iconically represents' is responsible for an enrichment of the logical semantics of count and mass terms. Furthermore, the iconic semantics is also responsible for the appearance of new discourse referents. Since so much of the theoretical burden is thus moved to the iconic component, future research will have to analyze its workings in much greater detail. (In addition, we have not tested examples in which mass terms allow for inferred discourse referents, and thus this point too will have to be investigated.)

\section{$9.2 \quad$ Improvements}

Our foregoing analyses might be overly influenced by the theory of English plurals, however ${ }^{41}$ As noted in Section 3.2.1, unrepeated ASL count terms can range over non-singleton groups. This observation makes it possible to develop a more unified analysis of plurals and mass terms (henceforth 'Improved Analysis 1') by taking unpluralized count terms to be true of atoms and non-atoms alike. On this revised view, (62)(i) could be simplified as in (63)(i) below (boldfaced to highlight the difference from (62)(i)). The simplification consists in eliminating the application of star operator * to count nouns.

(63) Improved Analysis 1: repetition as an iconic condition only: semantics of punctuated, unpunctuated and continuous repetitions without pluralization, but with the assumption that count terms are true of atoms and non-atoms

${ }^{41}$ Thanks to Malte Zimmermann (p.c.) for remarks that lead to these improvements. 


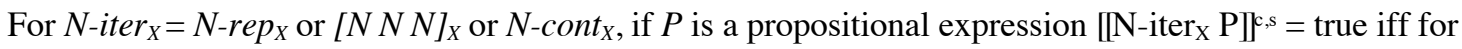
some group $\mathrm{d}$,

(i) $[\mathbb{N}]^{\mathrm{k}, \mathrm{s}[\mathrm{X} \rightarrow \mathrm{d}]}(\mathrm{d})=$ true, and

(ii) $N$-iter iconically represents $\mathrm{d}$ given $\mathrm{c}$, and

(iii) $[[\mathrm{P}]]^{\mathrm{c}, \mathrm{s}\left[\mathrm{X} \rightarrow \mathrm{d}, \mathrm{x}_{1} \rightarrow \mathrm{d}_{1}, \ldots, \mathrm{x}_{\mathrm{n}} \rightarrow \mathrm{d}_{\mathrm{n}}\right]}=$ true, where $x_{l}, \ldots, x_{n}$ are discourse referents made available by the iconic semantics of $N$-iter, and $x_{1}, \ldots, x_{n}$ respectively denote $\mathrm{d}_{1}, \ldots, \mathrm{d}_{\mathrm{n}}$ (which are parts of $\mathrm{d}$ ).

Importantly, on this alternative view the repetition does not change the type of denotation of the noun, which from the start holds true of atoms and non-atoms alike. Correspondingly, the clause in (63)(i) makes reference to $[[\mathrm{N}]]^{\mathrm{c}, \mathrm{s}[\mathrm{X} \rightarrow \mathrm{d}]}$ rather than to $*[[\mathrm{~N}]]^{\mathrm{c}, \mathrm{s}[\mathrm{X} \rightarrow \mathrm{d}]}$ as in (62)(i) above. On this view, then, the sole contribution of the repetition is to introduce an iconic condition (the iconic condition might then suffice to force the denotation of the variable $X$ to be a plurality ${ }^{42}$ ).

An alternative ('Improved Analysis 2'), suggested by Malte Zimmermann (p.c.), is to posit that unpluralized count terms are true of atoms and non-atoms alike (as in Improved Analysis 1), but that the repetition removes atoms from nominal extensions (if mass noun denotations lack atoms, the condition will be vacuous for them). This can be implemented by adding the boldfaced condition in (64)(i):

(64) Improved Analysis 2: repetition as an iconic condition with removal of atoms: semantics of punctuated, unpunctuated and continuous repetitions without pluralization, but with (i) the assumption that 'count' terms are true of atoms and non-atoms, and (ii) removal of atoms from the extension of repetitionbased constructions

For $N$-iter $r_{X}=N-r e p_{X}$ or $[N N N]_{X}$ or $N$-cont , $_{\text {, }} P$ is a propositional expression, $\left[\left[\mathrm{N} \text {-iter } \mathrm{X}_{\mathrm{P}} \mathrm{P}\right]\right]^{\mathrm{c}, \mathrm{s}}=$ true iff for some group $\mathrm{d}$,

(i) $\mathbf{d}$ is not an atom, and $[[\mathrm{N}]]^{\mathrm{c}, \mathrm{s}[\mathrm{X} \rightarrow \mathrm{d}]}(\mathrm{d})=$ true $^{43}$, and

(ii) $\mathrm{N}$-iter iconically represents $\mathrm{d}$ given $\mathrm{c}$, and

(iii) $[[\mathrm{P}]]^{\mathrm{c}, \mathrm{s}\left[\mathrm{X} \rightarrow \mathrm{d}, \mathrm{x}_{1} \rightarrow \mathrm{d}_{1}, \ldots, \mathrm{x}_{\mathrm{n}} \rightarrow \mathrm{d}_{\mathrm{n}}\right]}=$ true, where $x_{1}, \ldots, x_{n}$ are discourse referents made available by the iconic semantics of $\mathrm{N}$-iter, and $x_{1}, \ldots, x_{n}$ respectively denote $\mathrm{d}_{1}, \ldots, \mathrm{d}_{\mathrm{n}}$ (which are parts of $\mathrm{d}$ ).

If it turns out that a preferred singular reading arises in the absence of repetitions, this might be explained (in non-negative environments) by a competition with the repeated form by way of an implicature (possibly even a locally computed one). Specifically, if the repeated form BOOK-rep can be interpreted without an iconic condition, the utterance of unrepeated $B O O K$ in (65) a could trigger the implicature that (65)b is false. This is because for Improved Analysis 2, (65)b, which asserts the existence of a non-atomic object $d$ made of books, is more informative than (65)a, which just asserts the existence of an atomic or non-atomic object $\mathrm{d}$ made of books. ${ }^{44}$

(65) a. POSS-2 APARTMENT HAVE BOOK.

Predicted meaning for Improved Analysis 2,: Your apartment has a group of one or more books. b. POSS-2 APARTMENT HAVE BOOK-rep.

Predicted meaning for Improved Analysis 2: Your apartment has a group of at least two books.

When we turn to negative environments, Improved Analysis 2 makes potentially different predictions from the initial theory in (62) and from Improved Analysis 1. Consider (66)a,b:

\footnotetext{
${ }^{42}$ In this latter respect, the situation is similar in the initial account in (62). Without an iconic condition, (62) would allow a singular object d to satisfy the condition $*\left[\mathrm{~N}^{\mathrm{c}}\right]^{\mathrm{s}[\mathrm{X} \rightarrow \mathrm{d}]}(\mathrm{d})=$ true; this is because Link's star operator (defined in (29)a) yields a set of atoms and non-atoms.

${ }^{43}$ Since Improved Analysis 2 (like Improved Analysis 1) takes $N$ to hold true of both atoms and sums, $\mathrm{d}$ is specified to be a non-atomic sum. This corresponds to what Link 1998 calls a 'genuinely pluralic' $N$. For him (D. 37, p. 67), an object d is 'a genuinely pluralic' $N$ just in case $\mathrm{d}$ is a sum consisting of $N$ 's, but not an atom (in other places [Link 1998, D. 12, p. 23], Link talks of 'proper plural predicates' for predicates $N$ that are just true of such objects). Thanks to Malte Zimmermann for these references.

${ }^{44}$ Things are more complex if repeated forms systematically come with an iconic condition, for in that case (65)b might fail to be uttered because the signer lacks the relevant iconic information; this, in turn, would make the implicature triggered for (65)a more complicated.
} 
(66) a. POSS-2 APARTMENT NEVER HAVE BOOK-rep.

b. IF POSS-2 APARTMENT HAVE BOOK-rep, IX-1 1-GIVE-2 20 DOLLAR.

a'. Your apartment never has books.

b'. If your apartment has books, I'll give you $\$ 20$.

If $B O O K$-rep can be realized without an iconic contribution, the initial theory in (62) as well as Improved Analysis 1 predict the condition POSS-2 APARTMENT HAVE BOOK-rep to be satisfied as soon as there is a single book in the apartment (as is arguably the case for the English expressions in (66)a' and, less clearly, in (66)b'). In other words, in such a situation, (66)a should be false, while (66)b would entail that the signer must give $\$ 20$ to the addressee. Things are different on Improved Analysis 2, which predicts that a single book (i.e. an atom) could not satisfy $x$ HAVE BOOK-rep. Thus a 'single book' situation would not suffice to refute (66)a, nor to ensure that (66)b commits the signer to giving the addressee $\$ 20$.

As we noted in Section 3.2.2, we do not have evidence that repeated forms can range over singletons. In fact, in general they even come with a requirement that at least three objects should satisfy the relevant condition. This might initially seem to argue for Improved Analysis 2. But the issue is complex, because this 'at least two/at least three' condition might stem from the iconic condition rather than from the logical semantics of the repeated form. We leave this complex issue for future research.

\section{Towards a comparison with iconic gestures ${ }^{45}$}

In this section, we ask whether the inferences obtained with punctuated or unpunctuated repetitions can be replicated with gestures in spoken language (see Feldstein 2015 for an earlier attempt to study plurals in gesturers, and Schlenker to appear e for a discussion of 'homogeneity inferences' with gestural plurals). Specifically, we argue that (i) gestures with a nominal meaning can give rise to punctuated, unpunctuated and continuous repetitions, and that (ii) these repetitions come with comparable numerical threshold conditions as in ASL (we leave open whether the Edge Effect discussed in Section 6.1 can be replicated with gestures).

Following most of the recent literature on formal gesture semantics (e.g. Ebert and Ebert 2014, Schlenker to appear c, d Schlenker 2017b, to appear e), we base our analysis on introspective judgments of native informants pertaining to sentences they may never have seen before, and which may be very uncommon. While the introspective method has been argued to yield reliable results in linguistics (e.g. Sprouse and Almeida 2012), introspective judgments about gesture semantics should of course be validated with experimental means in the future. But we believe that this second step will be most fruitful if clear generalizations and theoretical claims have already been derived by way of introspective judgment (see Schlenker and Chemla, to appear, for a combined theoretical and experimental study of some word-replacing gestures that bear a resemblance to sign language agreement verbs).

While most of the literature has focused on co-speech gestures (produced at the same time as spoken expressions), gestures may also occur after the expressions they modify (henceforth 'postspeech gestures'), or they may replace words ('pro-speech gestures'; see for instance Kendon 2004, McNeill 2005, Giorgolo 2010, Schlenker to appear d for discussions of the typology of gestures, SlamaCazacu 1976, Clark 1996, Fricke 2008, Ladewig 2011 for pro-speech gestures, and Loehr 2004 for issues of intonation and alignment). Thus in all the sentences in (67), the addition of the gesture triggers the inference that the punishment was an objectionable action, involving a physical component. But in (67)a the gesture co-occurs with punish, in (67)b it follows the entire sentence, and in (67)c it fully replaces the verb.

Notation: A co-speech gesture is represented before the expression it modifies, and this expression is boldfaced. A post-speech gestures is represented after the expression it modifies, and is separated from

\footnotetext{
${ }^{45}$ Thanks to Sam Alxatib, Salvador Mascarenhas, Rob Pasternak, Brent Strickland and Lyn Tieu for discussion of some data discussed in this section.

(With apologies to the reader, we cite from the literature some examples - involving a slapping gesture - which refer to objectionable actions.)
} 
it by a dash (-) to represent a pause. A pro-speech gesture appears in lieu of a word. As in sign language, $I X$ transcribes a pointing sign/gesture.

(67) a. Co-speech gesture:

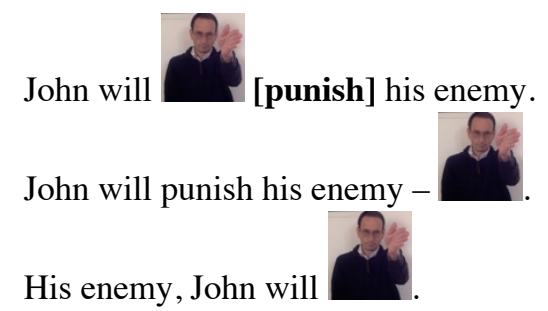

In recent semantic work (Schlenker to appear c, d), it was proposed that pro-speech gestures make at-issue contributions, that post-speech gestures contribute 'supplements' in the same way as appositive relative clauses, and that co-speech gestures introduce presuppositions of a special sort; examples are given in (68). Arguments for these conclusions involve the interaction between gestures and logical operators, and require complex examples that we cannot go into here. ${ }^{46}$

(68) a. Possible analysis of (67)a

At-issue contribution: John will punish his enemy.

Presupposition: If John punishes his enemy, slapping will be involved.

b. Possible analysis of (67)b

At-issue contribution: John will punish his enemy.

Supplementary contribution: This would involve some slapping.

$\approx$ John will punish his enemy, which will involve slapping him.

c. Possible analysis of (67)c

At-issue contribution: John will slap his enemy.

While these examples involve gestures with a verbal meaning, nominal gestures can be used as well, and they make it possible to replicate the main properties of punctuated, unpunctuated and continuous repetitions in ASL. The clearest case probably involves pro-speech gestures, with which we start our discussion. Let us consider the paradigm in (69), where it might help to produce underdetermined onomatopoeias (an informant tells us that iterations of $d a$ might be fairly natural). Here and throughout, the gesture for CROSS is based on the version illustrated in the picture, and as in our earlier sign language examples, the horizontal version involves repetition of the sign in a row, in front of the signer, with the shape ..., while the triangular version involves a vertical triangle with the tip above, with the shape $\therefore .^{47}$

\footnotetext{
${ }^{46}$ There are alternative accounts as well - in particular, Ebert and Ebert 2014 argue that co-speech gestures rather than post-speech gestures should be compared to supplements.

${ }^{47}$ See Schlenker to appear e for a discussion of the semantics of unrepeated CROSS, where it is argued that it is unmarked for number (= one or several crosses), but triggers 'exactly one' implicatures in unembedded clauses.
} 
(69) Context: The addressee is taking part in a treasure hunt in churches. The speaker provides an indication about the location of the treasure.

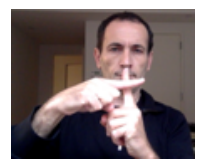

a. If you enter a room and you see [CROSS CROSS CROSS $]_{\text {hrizontal }}$, you have reached the prize. $\Rightarrow>$ if there are three crosses (arranged in a row?), the addressee has reached the prize b. If you enter a room and you see [CROSS CROSS CROSS $]_{\text {triangle }}$, you have reached the prize. $\Rightarrow>$ if there are three crosses arranged in a triangle, the addressee has reached the prize c. $[?]^{48}$ If you enter a room and you see CROSS-rep $3_{\text {horizontal }}$, you have reached the prize. $\Rightarrow$ if there are several crosses (arranged in a row ?), the addressee has reached the prize d. [?] If you enter a room and you see CROSS-rep3 $3_{\text {triangle }}$, you have reached the prize. $\Rightarrow>$ if there are several [or three?] crosses arranged in a triangle, the addressee has reached the prize e. [?] If you enter a room and you see CROSS-rep6 $6_{\text {horizontal }}$, you have reached the prize. $\Rightarrow>$ if there are lots of crosses (arranged in a row?), the addressee has reached the prize f. [?] If you enter a room and you see CROSS-rep6 $6_{\text {triangle }}$, you have reached the prize. $\Rightarrow$ if there are lots of crosses arranged in a triangle, the addressee has reached the prize

In all cases, the gestural contribution is interpreted within the conditional, and it does not lead to 'projection' phenomena characteristic of presuppositions and supplements. In particular, we do not derive an inference that if there are crosses in the room, they should be arranged in a particular way, or should be present in a certain number (this is the type of inference predicted by Schlenker to appear c, $\mathrm{d}$ for co-speech gestures co-occurring with noun such as crosses). In other words, the iconic properties of the pro-speech gesture appear to be at-issue. In addition, we believe that we might replicate some key properties of punctuated and unpunctuated repetitions in ASL:

- First, punctuated repetitions give rise to a precise reading, amounting to at least three in (69)a,b. By contrast, the threshold established by unpunctuated repetitions is not precise.

- Second, when unpunctuated repetitions involve more iterations, the threshold correspondingly goes up (but remains imprecise).

- Third, in all cases there is a clear iconic component when the iterations are arranged as a triangle. When they are arranged as a row, things might be a bit less clear, but we already observed a version of this in (48), which provided clearer iconic information upon the addition of a possessive targeting an edge. It is possible that a horizontal repetition is an iconically marked case, a point that should be investigated in future research. ${ }^{49}$

It would be desirable to test the existence of the Edge Effect (discussed for ASL in Section 6) with pro-speech plurals and pro-speech pointing. While some preliminary judgments suggest that it can be replicated, others do not bear this out, and thus the issue has to be investigated more systematically.

In the foregoing discussion, we followed Schlenker and Chemla (to appear) in taking prospeech gestures to offer a particularly good way of replicating some properties of signs within spoken language. We believe that co- and post-speech gestures could be used as well, but their interaction with logical operators would probably be more complicated - an issue we leave for future research. A simple paradigm without embedding is given in (70) (as a reminder, the gestures are encoded in capital letters before the expressions they modify, which are boldfaced).

(70) Context: The speaker is taking part in a treasure hunt, and he has been told to look for a cross

\footnotetext{
${ }^{48}$ We put [?] on (69)c,d,e,f to take into account a more formal survey (with videos and a written answer sheet) conducted with two informants after the present article was nearly completed. One informant (Informant 1) found all examples entirely acceptable, while the other (Informant 2) found (69)c,d,e,f degraded because the crosses were not clearly separated. In an inferential task in which 'three or more [imprecise]' corresponded to what is summarized in the example as 'several', Informant 2 still had the same type of inferential judgments as Informant 1 , except for the number of crosses involved in (69)d (Informant 1 took the requirement to the condition to involve 'three crosses or more [imprecise]', whereas Informant 2 leaned towards 'exactly three crosses [precise]').

${ }^{49}$ An anonymous referee suggests that the fact that the triangular shape is non-stereotypical might explain why this condition is at-issue; we leave this question too for future research.
} 
At last, I entered a room and I saw

Co-speech gestures:

a. CROSS-rep $3_{\text {row }}$ [crosses].

$\Rightarrow$ there were crosses arranged in a row

b. CROSS-rep $3_{\text {triangle }}$ [crosses].

$\Rightarrow$ there were crosses arranged in a triangle

Post-speech gestures:

c. crosses - CROSS-rep $3_{\text {row }}$.

$\Rightarrow$ there were crosses arranged in a row

d. crosses - CROSS-rep 3 triangle.

$\Rightarrow$ there were crosses arranged in a triangle

We believe that upon embedding under operators - such as an if-clause - it is far less clear that these co- or post-speech gestures can make an at-issue contribution (they are predicted not to by the theories developed in Schlenker to appear c, d). Since the topic of 'gesture projection' is outside the scope of the present paper, pro-speech gestures are a better choice to test the simplest semantic effects produced by punctuated and unpunctuated gestural repetitions.

Finally, it is our impression that some gestures can be used with a mass meaning, and that when this happens we replicate important aspects of ASL iconic mass terms. We attempted to do so in (71), where FLAT-HAND stands for a flat hand in the horizontal plane, making a small circular/trembling motion, and used to refer to areas of a disgusting substance. A continuous repetition is quite appropriate; and if a discontinuous repetition is used instead, it is understood that there were several disjoint parts of the relevant substance.$^{50}$ (In (71), a disgusted facial expression :-( co-occurs with the gesture; and it might help if in (71)c the disgusted expression is iterated three times, once with each gesture).

(71) There was a leak coming from the upstairs neighbor's bathroom, so when I got back home, I saw

a. :-( [FLAT-HAND-cont]

an area of a disgusting substance

b. :-( [FLAT-HAND-rep3]

several (?) puddles of a disgusting substance

c. :-( [FLAT-HAND FLAT-HAND FLAT-HAND]

three (?) puddles of a disgusting substance

While all the examples discussed in this section ought to be tested much more systematically (and if possible with experimental means), we believe that they suggest a fruitful comparison between iconic plurality in signs and in gestures.

\section{Conclusion}

Our investigations suggest that, in our consultant's ASL at least, repeated nouns can have plural and mass meanings, while simultaneously providing iconic information about the arrangement of the denoted group or substance. In our final analysis, a relatively unified semantics can be given for unpunctuated, punctuated and continuous repetitions (although some key theoretical choices are left for future research).

In each case, the shape of the sign provides at-issue iconic information about the arrangement of the denoted group or substance. Thus a punctuated repetition serves to refer to clearly separated objects, and it normally comes with a precise quantitative condition that corresponds to the number of iterations of the sign - unless the repeated noun co-occurs with a numeral. An unpunctuated repetition serves to refer to groups without imposing clear quantitative thresholds. This is not because an unpunctuated repetition imposes no quantitative condition at all, as can be seen by the fact that more (and possibly faster) repetitions refer to larger quantities. But unpunctuated repetitions are iconically vague: the precise number of repetitions is not presented as significant. A continuous repetition is primarily used for mass terms (with lexical constraints we do not understand), but it can to some extent

\footnotetext{
${ }^{50}$ An anonymous referee who generally agrees with our gestural judgments finds (71)b unnatural, however.
} 
be used with count nouns to refer to dense arrangements of objects. In addition, non-continuous repetitions can be used with mass terms, and they refer to a plurality of parts of the relevant substance.

Future research should explore the connection between repetition-based pluralities in ASL and in home signs. Recent studies of homesigners tend to group their punctuated repetitions with numerals because both give rise to precise quantitative conditions (Coppola et al. 2013, Abner et al. 2015). But if the present study is on the right track, one might expect that punctuated repetitions have more in common with unpunctuated repetitions than one might have initially thought: the difference between them might be primarily due to their iconic semantics rather than to a categorical grammatical difference.

In ASL, we found that punctuated and unpunctuated repetitions give rise to iconically inferred discourse referents denoting singular parts of the relevant groups. In simple cases, each iteration in a punctuated repetition yields a new discourse referent (although things might be more complex when a punctuated repetition co-occurs with a numeral). For unpunctuated repetitions, it is only edges of the repeated sign that yield new discourse referents. We suggested that this fact should follow from an appropriate iconic semantics: edges are presented as sharp representations whereas non-edges have lower resolution, and thus fail to refer by themselves. But a detailed understanding of this iconic semantics is left for future research, and our account of the edge effect is thus promissory at this point.

Strikingly, our data suggest that our standard logical semantics interacts in sophisticated ways with an iconic semantics, in particular in connection with anaphora - a point that was already made with respect to arc-based plural pronouns in Schlenker et al. 2013. This intricate interaction between iconic and logical semantics is hard to see in spoken language.

Hard, but not impossible: if we are right, pro-speech gestures might make it possible to replicate with gestures some of the iconic effects we saw at work with repeated signs. We believe that such prospeech gestures are very rare, but it is all the more striking that they seem to be readily understood, with fine-grained semantic distinctions that are reminiscent of sign language data. Still, the facts would need to be investigated far more systematically and if possible with experimental means (as in Schlenker and Chemla, to appear). One crucial difference is of course that gestures are not words, and thus come with severe expressive limitations, whereas all sorts of repeated signs can give rise to rich iconic modulations. We also noted that new singular discourse referents can be created by plural pro-speech gestures, but that these are best recovered either by pointing gestures alone, or by pointing gestures cooccurring with deictic elements such as that or that one.

Besides the development of a far more precise iconic semantics, this piece leaves several issues open. First, our ASL data should be tested with other nominal constructions and with other signers, and one should try to replicate them in other sign languages. Second, it would be interesting to compare repeated nouns with repeated pointing signs, which give rise to plural readings as well but can be contrasted with arc-based plural pronouns (for relevant remarks, see Kuhn 2015, Section 3.1). Third, our repetition-based plurals should be compared more closely with the repetition-based pluractionals studied in sign language by Kuhn and Aristodemo 2017, and in spoken language by Henderson 2016. An interesting point of comparison for the latter research might be obtained by studying cases in which an onomatopoeia is repeated to yield a plural nominal in spoken language. Fourth, the issue of mass terms that are 'countified' by iconic means (as in (9)c) should be investigated. Finally, our gestural data are very preliminary and ought to be investigated in greater depth. 
Supplementary Materials: Raw Data

Raw ASL data can be downloaded in .doc format at the following URL:

https://drive.google.com/file/d/1LJdokIdk80qvezHuG1Ji8dYaS ZGevpr/view 


\section{References}

Abner, N., Namboodiripad, S., Spaepen, E. \& Goldin-Meadow, S. (2015, January). Morphology in Child Homesign: Evidence from Number Marking. (Slides of a talk given at the 2015 Annual Meeting of the Linguistics Society of America, Portland, Oregon)

Abner, N. \& Wilbur, R. (to appear). Quantification in American Sign Language. (To appear in Edward Keenan and Denis Paperno (Eds.), Handbook of Quantifiers in Natural Language, Volume 2)

Chierchia, G. (2010). Mass nouns, vagueness and semantic variation. Synthese, 174, 99-149

Clark, H. H. (1996). Using language. (Cambridge: Cambridge University Press)

Coppola, M., Spaepen, E., \& Goldin-Meadow, S. (2013). Communicating about quantity without a language model: Number devices in homesign grammar. Cognitive Psychology, 67, 1-25

Daw, N. (2012) How vision works: The physiological mechanisms behind what we see. Oxford University Press, New York.

Deal, A. R. (2017). Countability distinctions and semantic variation. Natural Language Semantics 25, 125-171

Ebert, C. \& Ebert, C. (2014, June). Gestures, Demonstratives, and the Attributive/Referential Distinction. (Handout of a talk given at Semantics and Philosophy in Europe (SPE 7), Berlin)

Emmorey, K. and Herzig, M. (2003). Categorical versus gradient properties of classifier constructions in ASL. (In K. Emmorey (Ed.), Perspectives on Classifier Constructions in Signed Languages, Lawrence Erlbaum Associates, Mahwah NJ, pp. 222-246)

Feldstein, E. (2015). The development of grammatical number and space: Reconsidering evidence from child language and homesign through adult gesture. Short manuscript, Harvard University.

Fischer, S. (1973). Two Processes of Reduplication in the American Sign Language. Foundations of Language, 9: 469-480.

Fricke, E. (2008). Grundlagen einer multimodalen Grammatik des Deutschen: Syntaktische Strukturen und Funktionen. Habilitation treatise, European University Viadrina, Frankfurt (Oder)

Giorgolo, G. (2010). Space and Time in Our Hands. PhD Dissertation, Utrecht University

Greenberg, G. (2013). Beyond Resemblance. Philosophical Review, 122, 2

Henderson, R. (2016). Pluractional Demonstrations. Manuscript. http://ling.auf.net/lingbuzz/003127

Heim, I. \& Kratzer, A. (1998). Semantics in Generative Grammar. (Blackwell)

Ionin, T. \& Matushansky, O. (2006). The composition of complex cardinals. Journal of Semantics, 23, $315-360$

Jenkins, R. \& Kerr, C. (2013). Identifiable images of bystanders extracted from corneal reflections.

PLoS One. 8(12):e83325. DOI 10.1371/journal.pone.0083325

Kendon, A. (2004). Gesture: visible action as utterance. (Cambridge)

Koulidobrova, E. (2018). Counting Nouns in ASL. Manuscript, Central Connecticut State University. http://ling.auf.net/lingbuzz/003871

Kuhn, J. (2015). Cross-categorial singular and plural reference in sign language. Doctoral dissertation, New York University

Kuhn, J. \& Aristodemo, V. (2017). Pluractionality, iconicity, and scope in French Sign Language. Semantics \& Pragmatics 10(6).

Ladewig, S. (2011). Syntactic and semantic integration of gestures into speech: Structural, cognitive, and conceptual aspects. $\mathrm{PhD}$ thesis, Frankfurt (Oder): European University Viadrina

Link, G. (1998). Algebraic Semantics in Language and Philosophy. (Stanford: CSLI)

Lima, S. (2014, May) All notional mass nouns are count nouns in Yudja. (Proceedings of SALT 24, 534-554)

Loehr, D. P. (2004). Gesture and intonation. Doctoral dissertation, Georgetown University, Washington, DC

McNeill, D. (2005). Gesture and Thought. (University of Chicago Press)

Nicolas, D. (2016). The Logic of Mass Expressions. The Stanford Encyclopedia of Philosophy (Winter 2016 Edition), Edward N. Zalta (ed.), <https://plato.stanford.edu/archives/win2016/entries/logicmassexpress/>

Nijhof, S. \& Zwitserlood, I. (1999). Pluralization in Sign Language of the Netherlands (NGT). (In Jan Don \& T. Sanders (eds.), OTS Yearbook 1998-1999, (pp. 58-78). Utrecht: Utrechts Instituut voor Linguistiek OTS) 
Nouwen, R. (2003). Plural pronominal anaphora in context. (Number 84 in Netherlands Graduate School of Linguistics Dissertations, LOT, Utrecht)

Nouwen, R. (2015). Plurality. (In: P. Dekker and M. Aloni (eds), Cambridge Handbook of Semantics. Cambridge University Press)

Petronio, K. (1995). Bare Noun Phrases, Verbs and Quantification in ASL. (In E. Bach, E. Jelinek, A. Kratzer \& B. Partee (Eds.), Quantification in Natural Language (Vol. 2, pp. 603 - 618). Kluwer Academic Publishers)

Pfau, R. \& Steinbach, M. (2006). Pluralization in sign and in speech: A cross-modal typological study. Linguistic Typology, 10, 49-135

Sandler, W. \& Lillo-Martin, D. (2006). Sign Language and Linguistic Universals. (Cambridge University Press)

Sauerland, U. (2003, May). A new semantics for number. (In R. Young \& Y. Zhou (Eds.), Proceedings of SALT 13. Ithaca, NY: CLC Publications, Cornell University)

Schlenker, P. (2011). Donkey Anaphora: the View from Sign Language (ASL and LSF). Linguistics and Philosophy, 34(4), 341-395

Schlenker, P. (2014). Iconic Features. Natural Language Semantics 22, 4, 299-356

Schlenker, P. (2017a). Sign Language and the Foundations of Anaphora. Annual Review of Linguistics, 3, 149-77

Schlenker, P. (2017b). Gestural Grammar. Manuscript, Institut Jean-Nicod and New York University.

Schlenker, P. (to appear a). Visible Meaning: Sign Language and the Foundations of Semantics. (Accepted for publication as a target article in Theoretical Linguistics)

Schlenker, P. (to appear b). Super Monsters II. Semantics \& Pragmatics

Schlenker, P. (to appear c). Gesture Projection and Cosuppositions. Linguistics \& Philosophy

Schlenker, P. (to appear d). Iconic Pragmatics. Natural Language \& Linguistic Theory

Schlenker, P. (to appear e). Gestural Semantics: Replicating the typology of linguistic inferences with pro- and post-speech gestures. Natural Language \& Linguistic Theory.

Schlenker, P. \& Chemla, E. (2018). Gestural Agreement. Natural Language \& Linguistic Theory. 36, 2: 87-625587. https://doi.org/10.1007/s11049-017-9378-8

Schlenker, P., Lamberton, J. \& Santoro, M. (2013). Iconic Variables. Linguistics \& Philosophy 36(2), 91-149

Slama-Cazacu, T. (1976). Nonverbal components in message sequence: "Mixed syntax." (In W. C. McCormack \& S. A. Wurm (Eds.), Language and Man: Anthropological Issues (pp. 217-227). The Hague: Mouton)

Spector, B. (2007). Aspects of the pragmatics of plural morphology: on higher-order implicatures. (In U. Sauerland and P. Stateva. (Eds.), Presuppositions and Implicatures in Compositional Semantics, (pp 243-281). New York: Palgrave-Macmillan)

Sprouse, J. \& Almeida, D. (2012). Assessing the reliability of textbook data in syntax: Adger's Core Syntax. Journal of Linguistics, 48(3), 609-652

Supalla, T. (1982). Structure and Acquisition of Verbs of Motion and Location in American Sign Language. PhD Thesis, University of California, San Diego

Wilbur, R. (1987). American Sign Language: Linguistic and Applied Dimensions. (Boston: Little, Brown)

Zweig, E. (2006). The Implications of Dependent Plural Readings. (In: Davis, C., Deal, A. R.\& Zabbal, Y. (eds.). Proceedings of the thirty-sixth annual meeting of the North East Linguistic Society, p. 735)

Zwitserlood, I. (2012). Classifiers. (In Pfau, R., M. Steinbach \& B. Woll (eds.), Sign language. An international handbook. (pp 158-186). Berlin: De Gruyter Mouton) 